ARTICLE

DOI: $10.1038 / s 41467-017-01279-9$

\title{
Structure of SgK223 pseudokinase reveals novel mechanisms of homotypic and heterotypic association
}

Onisha Patel ${ }^{1,2}$, Michael D.W. Griffin ${ }^{3}$, Santosh Panjikar (10) 4,5, Weiwen Dai ${ }^{1,2}$, Xiuquan Ma ${ }^{5,6}$, Howard Chan ${ }^{5,6}$, Celine Zheng ${ }^{1,2}$, Ashleigh Kropp ${ }^{1,2}$, James M. Murphy ${ }^{1,2}$, Roger J. Daly ${ }^{5,6}$ \& Isabelle S. Lucet (iD ${ }^{1,2}$

The mammalian pseudokinase SgK223, and its structurally related homologue SgK269, are oncogenic scaffolds that nucleate the assembly of specific signalling complexes and regulate tyrosine kinase signalling. Both SgK223 and SgK269 form homo- and hetero-oligomers, a mechanism that underpins a diversity of signalling outputs. However, mechanistic insights into SgK223 and SgK269 homo- and heterotypic association are lacking. Here we present the crystal structure of SgK223 pseudokinase domain and its adjacent $\mathrm{N}$ - and C-terminal helices. The structure reveals how the $\mathrm{N}$ - and $\mathrm{C}$-regulatory helices engage in a novel fold to mediate the assembly of a high-affinity dimer. In addition, we identified regulatory interfaces on the pseudokinase domain required for the self-assembly of large open-ended oligomers. This study highlights the diversity in how the kinase fold mediates non-catalytic functions and provides mechanistic insights into how the assembly of these two oncogenic scaffolds is achieved in order to regulate signalling output.

\footnotetext{
${ }^{1}$ The Walter and Eliza Hall Institute of Medical Research, Parkville, VIC 3052, Australia. ${ }^{2}$ Department of Medical Biology, University of Melbourne, Parkville, VIC 3052, Australia. ${ }^{3}$ Department of Biochemistry and Molecular Biology, Bio21 Molecular Science and Biotechnology Institute, University of Melbourne, Parkville, VIC 3052, Australia. ${ }^{4}$ Australian Synchrotron, Clayton, VIC 3168, Australia. ${ }^{5}$ Department of Biochemistry and Molecular Biology, Level 1, Building 77, Monash University, Clayton, VIC 3800, Australia. ${ }^{6}$ Cancer Program, Biomedicine Discovery Institute, Monash University, Clayton, VIC 3800, Australia. Roger J. Daly and Isabelle S. Lucet contributed equally to this work. Correspondence and requests for materials should be addressed to

O.P. (email: patel.o@wehi.edu.au) or to I.S.L. (email: lucet.i@wehi.edu.au)
} 
P seudokinases have recently emerged as crucial regulators of many cellular functions. Despite a lack of catalytic activity, the pseudokinase (PsK) domain structure resembles that of classical kinases, enabling pseudokinases to undergo protein-protein interactions to disseminate or coordinate signal outputs. Their functions vary from allosteric regulators that finetune kinase catalytic activities ${ }^{1-6}$ to molecular switches that propagate signal output ${ }^{7}$ and scaffolds that nucleate the assembly of signalling complexes ${ }^{8-11}$.

Sugen kinase 223 (SgK223) (also known as Pragmin) and SgK269 (also known as PEAK1) are closely related proteins of 149 and $193 \mathrm{kDa}$, respectively. Both are classified as pseudokinases due to the substitution of critical residues within highly conserved motifs known to be essential for kinase activity ${ }^{12,13}$, and both are predicted to function as scaffolds ${ }^{10,14-16}$. They share a domain organisation that consists of an $\mathrm{N}$-terminal region predicted to be partly folded, a largely unstructured central PEST region and a Cterminal PsK domain flanked either side by predicted helical regulatory domains ${ }^{10}$. The PEST central region harbours tyrosine phosphorylation sites that specifically recruit $\mathrm{SH} 2$ and $\mathrm{PTB}$ domain-containing effectors, however the function of the conserved $\mathrm{N}$ - and $\mathrm{C}$-terminal regions, including the PsK domain, remains largely unknown.

SgK223 was first identified as an effector of Rnd2, a member of the Rho family of GTPases, and shown to regulate neuron outgrowth by functioning as a RhoA activator ${ }^{17}$. More recently, SgK223 has emerged as a protein scaffold that promotes Src family kinase (SFK) signalling by regulating the subcellular localisation of C-terminal Src kinase (Csk), a negative regulator of these kinases ${ }^{14}$. Once phosphorylated on tyrosine residue Y411, SgK223 binds and sequesters $\mathrm{Csk}^{14}$, which prevents Cskmediated SFK phosphorylation and inactivation at the plasma membrane, leading to sustained SFK activation. A recent study found SgK223 and Csk were co-localized at focal adhesions where they regulate cell morphology and motility ${ }^{18}$. Consistent with these findings, SgK223 has emerged as an important regulator of cell morphology, migration and invasion. Overexpression of SgK223 leads to a more elongated, fibroblastic morphology ${ }^{18}, 19$ and enhances migratory and invasive potential ${ }^{19}$. In addition, SgK223 is implicated in progression of specific cancers, being required for Src-induced invasion of colon cancer cells ${ }^{20}$, and upregulated during pancreatic cancer development, where it acts a positive regulator of Stat $3^{19}$.

The related protein, SgK269/PEAK1, is also implicated in regulation of cell migration. SgK269 localises to the pseudopodia of migrating cells, associates with the actin cytoskeleton and focal adhesions and promotes cell motility ${ }^{15}$. It plays a key role in regulating the maturation and disassembly of focal adhesions, a function that is dependent on SgK269 phosphorylation at Y665 and SFK activity ${ }^{21}$. During EGF signalling via the scaffold Shc1, SgK269 mediates a critical switch in signal output, binding Shc1 via SgK269 Y1188 and promoting migratory/invasive responses ${ }^{16}$. However, SgK269 can also promote mitogenesis by recruiting Grb2 to Y635 and activating the Ras pathway ${ }^{22}$. Reflecting these different functional properties, overexpression of SgK269 in mammary epithelial cells promotes a partial epithelial-mesenchymal transition, and aberrant growth and morphogenesis 22 . As with SgK223, SgK269 is implicated in human malignancy and is overexpressed in a subset of breast, colon and pancreatic cancers ${ }^{15,22,23}$.

In a recent study, we demonstrated that SgK269 and SgK223 are both capable of homo-dimerization, an event in part driven by a 43 amino-acid helix (termed $\alpha \mathrm{N} 1$ ) directly upstream of the PsK domain ${ }^{10}$. We also reported hetero-oligomerization between SgK223 and SgK269, a mechanism that is dependent on the presence of the $\alpha \mathrm{N} 1$ helix and the C-terminal containing PsK region and demonstrated the importance of SgK223-SgK269 hetero-oligomerization in promoting cell migration and STAT3 activation ${ }^{0}$. However, the molecular basis for these interactions has remained unclear. To gain further insight into the molecular mechanism that drives SgK223 dimerization and oligomerization functions, we solved the crystal structure of the PsK domain of SgK223 and its adjacent regulatory domains. Our structure reveals that the $\alpha \mathrm{N} 1$ helix and the C-terminal domain fold together in a unique helix bundle that drives SgK223 homodimerization. In addition, our structure uncovers an unexpected feature of the SgK223 PsK domain where the $\alpha \mathrm{G}$ helix forms a critical interaction interface that promotes oligomerization. Together, our structural data, combined with mutational analysis, biochemical and biophysical and cellular studies, provide a detailed understanding of the mechanism that drive homo- and heterotypic association of these two pseudokinase scaffolds, and suggests the importance of higher-order scaffold assembly in regulating signal output.

\section{Results}

SgK223 dimerizes via the PsK domain-flanking regions. To fully understand the structural determinants that drive $\mathrm{SgK} 223$ dimerization, we solved the crystal structure of a construct (SgK223- $\alpha$ N1-PsK-Cter) that encompasses the PsK domain and the adjacent $\mathrm{N}$ - and C-regulatory domains (Fig. 1a) using the multi-wavelength anomalous dispersion method. The structure was refined against native data to $2.95 \AA$ resolution. SgK223 crystallized in space group $\mathrm{P}_{3}{ }_{2} 2$ with one molecule in the asymmetric unit (Table 1). Within a monomer, the PsK domain of SgK223 adopts a typical kinase-like fold and the N-terminal helix $\alpha \mathrm{N} 1$ directly interacts with the C-terminal helices $\alpha \mathrm{J}$ and $\alpha \mathrm{K}$, while $\alpha \mathrm{L}$ helix slides under $\alpha \mathrm{I}$ helix of the PsK domain (Fig. 1b) revealing an unusual 'XL'-shaped helix bundle. While the asymmetric unit contains a monomer, the $\mathrm{P} 4_{3} 2{ }_{1} 2$ crystal form revealed a two-fold symmetric dimer with an extensive buried surface area (BSA) of $1620 \AA^{2}$. SgK223 dimer formation is mediated by the regulatory helices from the two monomers with the $\alpha \mathrm{N} 1$ and the $\alpha \mathrm{J}$ helices from one monomer interacting with the corresponding $\alpha \mathrm{J}$ and $\alpha \mathrm{N} 1$ helices from the second monomer to form a unique ' $\mathrm{XX}$ '-shaped four-helix bundle at the 'core' of the dimer and positioning the PsK domains peripherally to form the 'shell' of the dimer (Fig. 1c). This distinctive $\alpha \mathrm{N} 1 / \alpha \mathrm{J}$ helix bundle reveals an extensive interaction footprint consistent with a large BSA and highlights the rigidity of the dimeric packing (Fig. 1c and Supplementary Fig. 1). Within the 'XX' fold, $\alpha \mathrm{N} 1$ and $\alpha J$ arrange in a left-handed parallel fashion reminiscent of the arrangement of helices of leucine zipper GCN4, packing against each other in a 'knobs-into-holes' arrangement, further accentuating the shape complementarity of the dimeric interface (Supplementary Fig. 1). This packing is consistent with our previous data showing that the removal of $\alpha \mathrm{N} 1$ leads to a monomeric form of $\operatorname{SgK} 223^{10}$.

To further confirm the dimerization state of SgK223, we assessed the behaviour of SgK223 using analytical ultracentrifugation. Sedimentation velocity experiments with SgK223- $\alpha$ N1PsK-Cter at concentrations between 0.6 and $74.0 \mu \mathrm{M}$ indicated that at concentrations below $2.3 \mu \mathrm{M}$ the protein sedimented as a reasonably homogeneous species with a sedimentation coefficient of $\sim 5.6 \mathrm{~s}$ (Fig. 1d and Supplementary Fig. 1), consistent with a dimer. Weight average sedimentation coefficients calculated for $0.6,1.2$ and $2.3 \mu \mathrm{M}$ of SgK223- $\alpha \mathrm{N} 1-\mathrm{PsK}-\mathrm{Cter}$ were $5.55,5.62$ and $5.66 \mathrm{~s}$, corresponding to molecular weights (MW) of 120,113 and $96.5 \mathrm{kDa}$, respectively, provided further evidence for a dimeric structure in solution (monomer $\mathrm{MW}, 54 \mathrm{kDa}$ ). The relative stability of the weight average sedimentation coefficient over this 
concentration range suggests that the dimer $>$ monomer dissociation constant is in the mid- to low-nanomolar range. In contrast, at concentrations above $4.6 \mu \mathrm{M}$, the $c\left(s_{20, \mathrm{w}}\right)$ distributions shifted towards higher sedimentation coefficients, with a weight average sedimentation coefficient of $7.83 \mathrm{~s}$ at $74.0 \mu \mathrm{M}$, consistent with formation of tetramer and higher-order oligomers

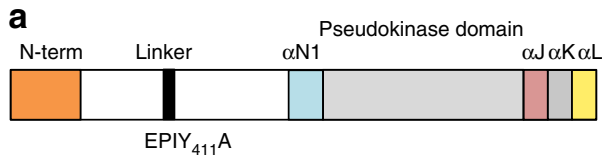

b

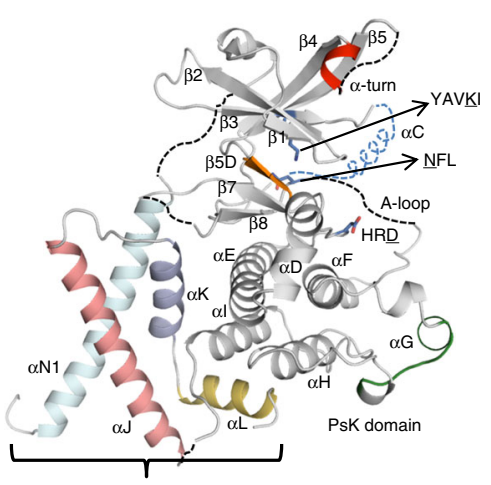

'XL'-shaped helix bundle

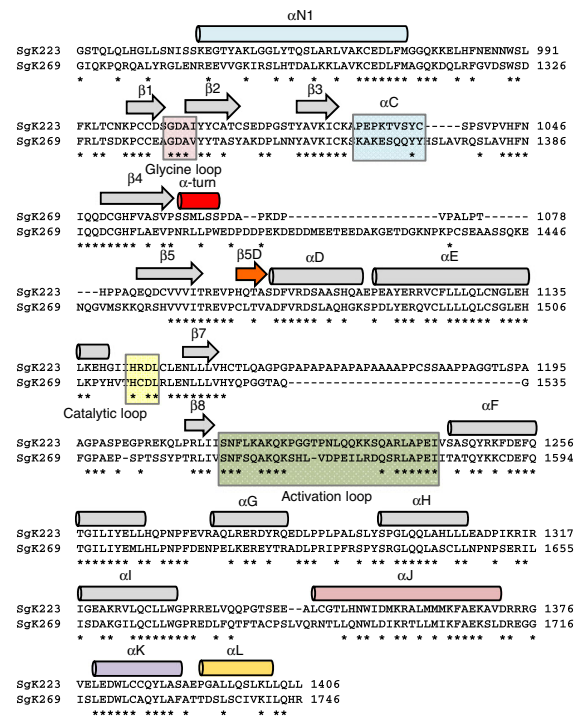

C

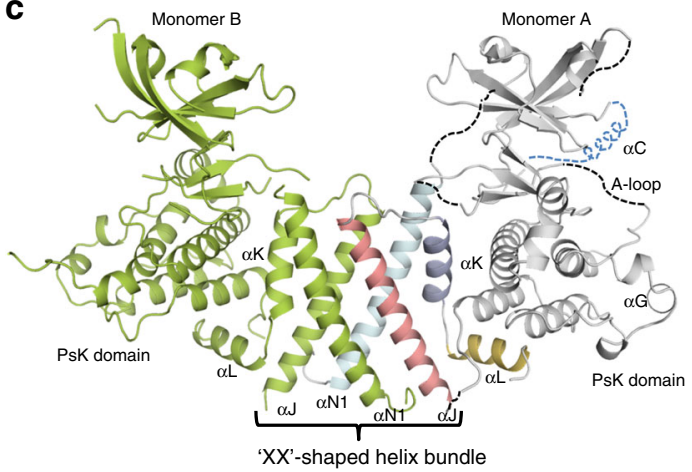

d SgK223- $\alpha$ N1-PsK-Cter

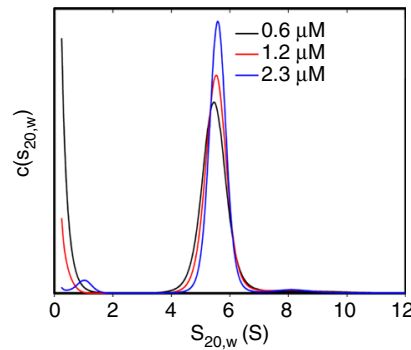

e

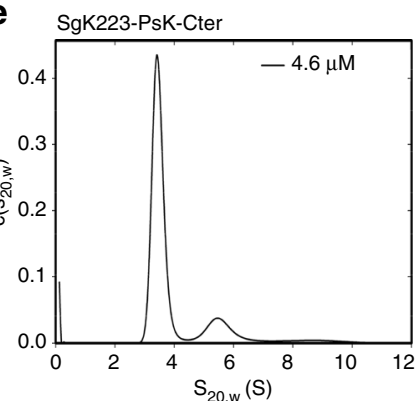

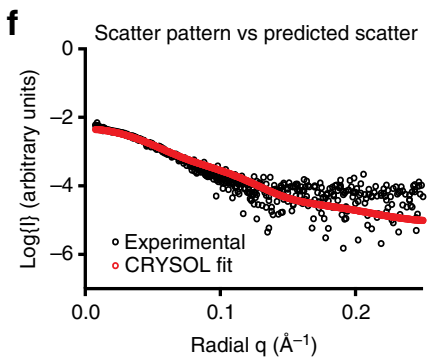
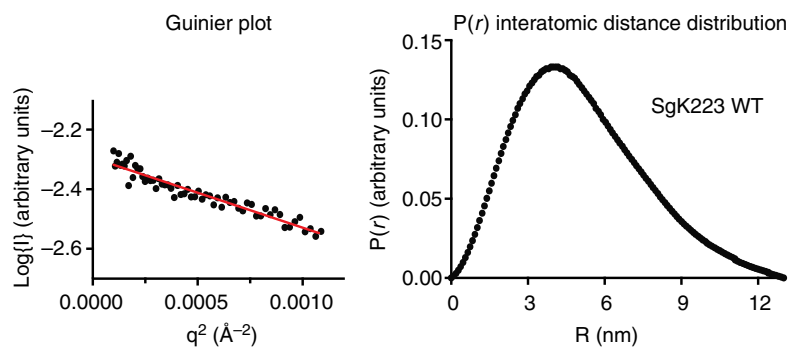

Fig. 1 Structure of SgK223- $\alpha$ N1-PsK-Cter. a Schematic representation of SgK223. Y411 is phosphorylated by Csk, and also serves as docking site for this kinase. $\mathbf{b}$ Structure of SgK223- $\alpha$ N1-PsK-Cter monomer (top) and sequence alignment of SgK223 and SgK269 and secondary structure elements as determined from the SgK223- $\alpha$ N1-PsK-Cter crystal structure (bottom). $\alpha$ N1, cyan; PsK domain, grey; $\alpha$ J, salmon; $\alpha \mathrm{K}$ violet; $\alpha \mathrm{L}$ yellow; catalytic residues are shown in sticks and underlined; $\alpha \mathbf{C}$ is modelled in blue dashed lines. c Structure of SgK223- $\alpha$ N1-PK-Cter dimer. Monomer A colour coding as in $\mathbf{b}$; monomer $B$ shown in green. $\mathbf{d}$ Continuous standardized sedimentation coefficient $\left[c\left(s_{20, w}\right)\right]$ distributions for SgK223- $\alpha$ N1-PsK-Cter at concentrations of $0.6 \mu \mathrm{M}$ (black line), $1.2 \mu \mathrm{M}$ (red line) and $2.3 \mu \mathrm{M}$ (blue line). e $c\left(s_{20, w}\right)$ distribution for SgK223-PsK-Cter at a concentration of $4.6 \mu \mathrm{M}$. $\mathbf{f}$ Left: overlay of experimental scattering data (black circles) and scattering profile calculated using CRYSOL (red). Middle: Guinier plot indicating that aggregates do not measurably contribute to the scattering profile. Right: Interatomic distance distributions. See also Supplementary Fig. 1 


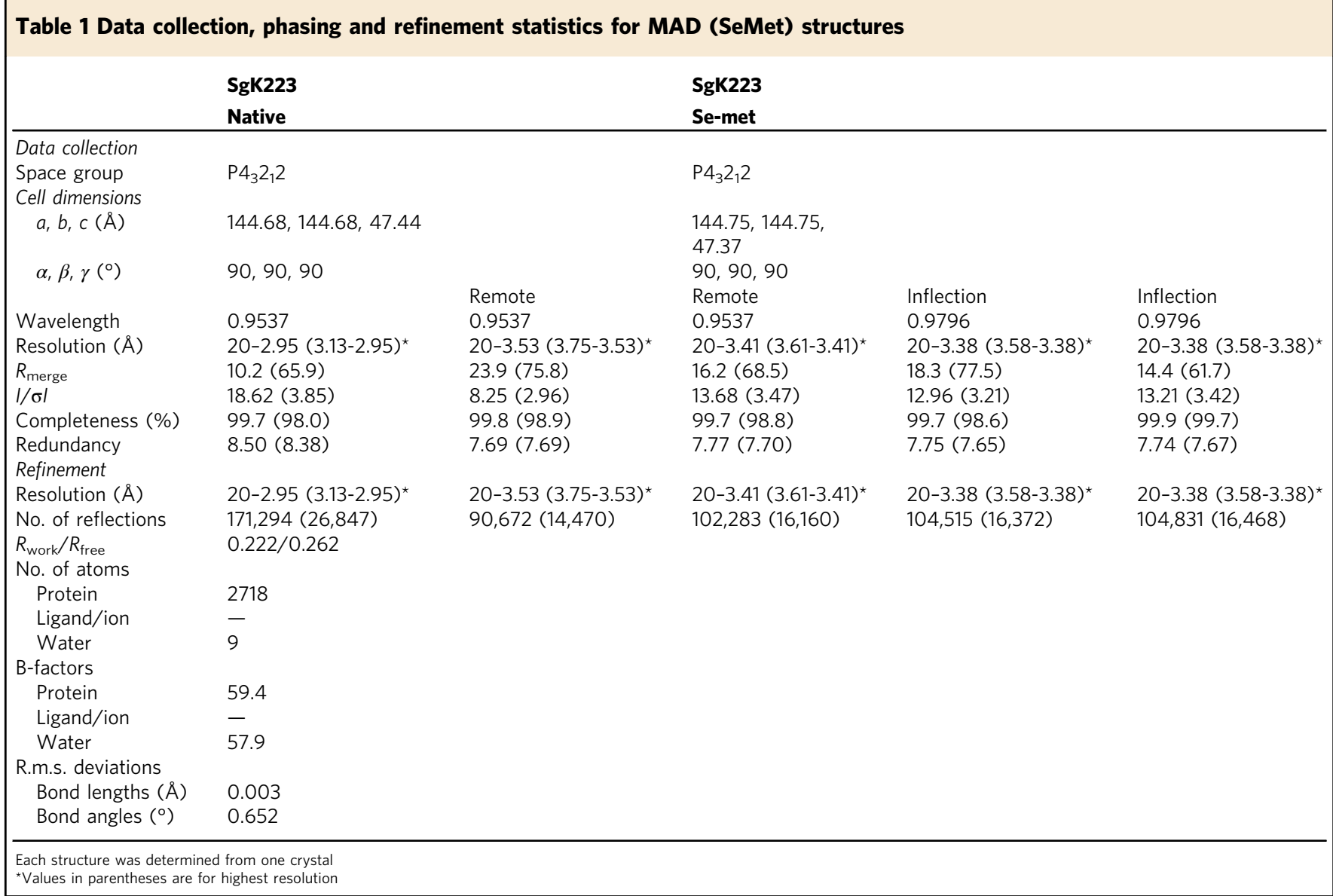

(Supplementary Fig. 1). Sedimentation velocity experiments with SgK223-PsK-Cter construct without $\alpha \mathrm{N} 1$, provided $c\left(s_{20, \mathrm{w}}\right)$ distributions with a major peak around $3.4 \mathrm{~s}$ (Fig. 1e) consistent with a monomer and our previous data showing that this construct eluted as a monomer by analytical size-elution chromatography $(\mathrm{SEC})^{10}$.

To verify that the homodimeric conformation observed in the crystal is representative of the in solution structure of SgK223, we performed Small-Angle X-ray Scattering (SAXS). The maximum particle dimension $\left(D_{\max }\right)$ was measured as $130 \AA$, consistent with the dimensions of the SgK223 dimer crystal structure. Furthermore, the experimental scatter pattern was consistent with the theoretical scatter calculated based on the crystal structure coordinates (Fig. 1f; $\chi=0.43$ ), thereby verifying the crystallized dimer is akin to the in solution conformation.

Taken together, the AUC and SAXS data confirm that SgK223 exists as a dimer in solution, validating the crystallized dimer conformation and confirming the structural role of regulatory helices in stabilizing the dimer interface.

SgK223 pseudokinase domain has unique features. The PsK domain of each monomer adopts a canonical bilobal kinase fold, which consists of an $\mathrm{N}$-lobe composed of five stranded $\beta$-sheets $(\beta 1-\beta 5)$ that connect to a larger $\alpha$-helical C-lobe through a hinge region. Despite a common overall kinase architecture fold, striking structural differences are seen within the $\mathrm{N}$-lobe. It contains a $\alpha$-turn (SSMLS) between the $\beta 4$ and $\beta 5$ strands, a feature also seen in elF $2 \alpha$ kinases $^{24}$, despite a lack of sequence similarity (Fig. $1 \mathrm{~b}$ and Supplementary Fig. 2). This $\alpha$-turn marks the start of an insertion loop unique to SgK223 and SgK269, however, its electron density is not resolved and its structural role remains unclear. In addition, the $\mathrm{N}$-lobe has an extra $\beta 5 \mathrm{D}$ strand that lies parallel to the $\beta 7$ and $\beta 8$ strands and connects the $\beta 5$ strand to the $\alpha \mathrm{D}$ helix (Fig. 1b). The most notable feature is the absence of a structured $\alpha C$ helix, a critical feature in bona fide kinases. The predicted $\alpha \mathrm{C}$ helix sequence in $\mathrm{SgK} 223$ is shorter and poorly conserved (Fig. 1b and Supplementary Fig. 2). Particularly, the $\alpha \mathrm{C}$ helix is missing the conserved glutamate, which forms the canonical $\beta 3$-Lys/ $\alpha \mathrm{C}$-Glu salt-bridge interaction, a structural feature of a catalytically active kinase conformation. Instead, in SgK223, the conserved $\beta 3$ Lys1024 from the YAVK motif makes H-bond interactions with Gln1048 from the loop preceding the $\beta 4$ strand. Gln1048 in turn makes H-bond interaction with Tyr1008 from the $\beta 2$ strand (Fig. $2 \mathrm{a}$ ) and the predicted gate-keeper residue Thr1092. This unusual interaction locks the orientation of the N-lobe of the PsK domain with respect to the C-lobe, clearly influencing the relative positions of the key residues known to be crucial for catalytic activity in conventional protein kinases. The N-lobe is further stabilized in this position by $\pi$-stacking interactions mediated by the triad of Phe1045 from the $\beta 3-\beta 4$ loop with Trp1382 from the $\alpha \mathrm{K}$ and Phe974 of $\alpha \mathrm{N} 1$ helix (Fig. $2 \mathrm{a}$ ). The $\beta 3$ - $\beta 4$ loop provides therefore a critical anchor point for the PsK domain to the $\mathrm{N}$ - and C-terminal regulatory domains. The start of the activation loop (A-loop) is characterized by SNF instead of the canonical DFG motif known to be crucial for the coordination of cations in bona fide kinases $^{25}$ (Fig. 2b). The SNF and the APE motifs are the only portion of the A-loop that are resolved in the structure. The catalytic loop $\left(\mathrm{HRD}_{1445} \mathrm{LCLEN}\right)$ adopts a conformation similar to bona fide kinases (Figs. 1b and 2a). The conserved tryptophan, Trp1330, at the end of the PsK domain, packs against the backbone of the $\alpha \mathrm{K}$ helix and the loop that connects the end of the 
a

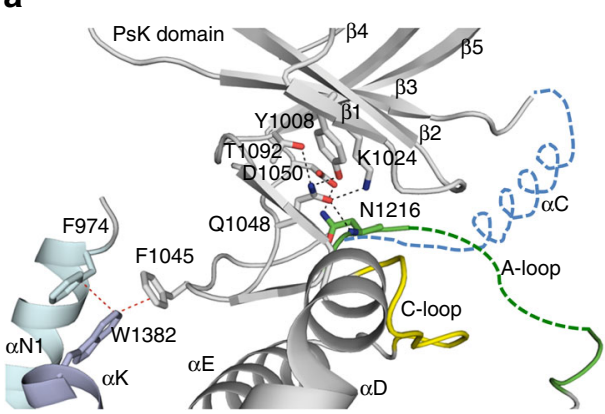

b

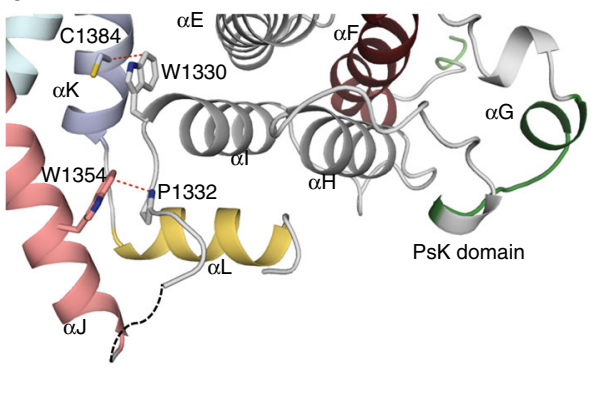

C

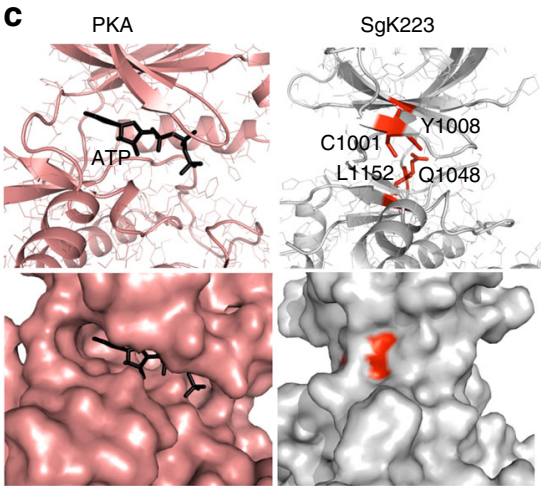

d

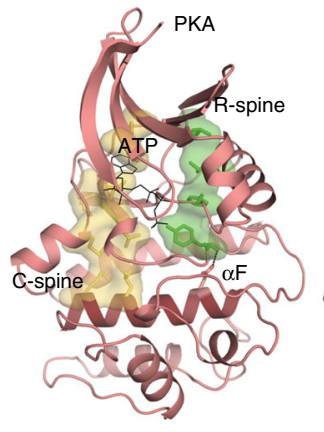

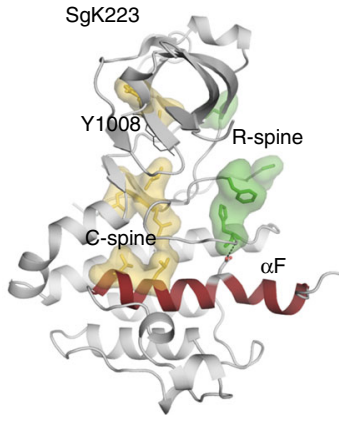

Fig. 2 The unique features of SgK223 PsK domain. a Interaction between N-lobe of the PsK domain and the dimerization domain. $\mathbf{b}$ Interaction between the C-lobe of the PsK domain and the dimerization domain. $\alpha \mathrm{N1}$, cyan; PsK domain, grey; $\alpha$ J, salmon; $\alpha \mathrm{K}$ violet; $\alpha \mathrm{L}$ yellow; A-loop, green; C-loop, yellow; $\alpha \mathrm{C}$ is modelled in blue dashed lines. c Comparison of the nucleotide binding site of PKA (left) and SgK223 (right). PKA, salmon; ATP, black; SgK223, grey, residues occupying the ATP-binding sites shown in red. $\mathbf{d}$ Location of $\mathrm{C}$ - and R-spines on PKA and SgK223. C-spine, yellow; R-spine, green; $\alpha F$, dark red

PsK domain and the start of the C-terminal helices. This interaction is stabilized by the bulky side chain of Trp1354 from the $\alpha \mathrm{J}$ helix, keeping the C-lobe of the PsK domain tightly anchored to the dimerization domain (Fig. 2b).

SgK223 has a degraded ATP-binding pocket. We previously determined by thermal shift assay (TSA) that SgK223 did not detectably bind nucleotides ${ }^{12}$. The structure of SgK223 clearly demonstrates an absence of an ATP-binding cavity, which is instead occupied by the side chains of residues Tyr1008, Cys1001, Gln1048 and Leu1152 (Fig. 2c). The hydrophobic interactions between Phe1045 ( $\beta 3-\beta 4$ loop, N-lobe PsK), Trp1382 $(\alpha \mathrm{K})$ and Phe $974(\alpha \mathrm{N} 1)$, the interaction between Trp1354 $(\alpha \mathrm{J})$ and Pro1332 (C-lobe PsK) and the triad interaction between Lys1024 $(\beta 3)$, Gln1048 ( $\beta 3-\beta 4$ loop) and Tyr1008 ( $\beta 2)$ all highly contribute to the rigidity of the positioning of the $\mathrm{N}$-lobe relative to the C-lobe and the loss of the ATP binding site (Fig. 2a). As a result of these modifications, the critical residues that make up the regulatory (R-) and catalytic (C-) spines are no longer aligned in SgK223 when compared to the canonical, active structure of the archetypal kinase PKA, suggesting a severely impaired catalytic core (Fig. 2d). Interestingly, the residues that occupy the ATP binding cavity in SgK223 are conserved in SgK269 (Fig. 1b) predicting that SgK269 is also devoid of any nucleotide binding ability, consistent with our earlier findings using TSA ${ }^{12}$.

The adjacent helices dictate SgK223 PsK domain conformation. Within a monomer of SgK223, the regulatory helices $\alpha \mathrm{N} 1$, $\alpha \mathrm{J}$ and $\alpha \mathrm{K}$ are stabilized by a number of polar and non-polar interactions. Importantly, $\alpha \mathrm{J}$ Phe1366 makes direct interactions with $\alpha \mathrm{K}$ Asp1381 and Cys1385 and is buried in a hydrophobic pocket formed by neighbouring Met1362 and Val1377 located in the $\alpha \mathrm{J}-\alpha \mathrm{K}$ connecting loop, and $\alpha \mathrm{N} 1 \mathrm{Cys} 970$ and Leu966. In addition, $\alpha \mathrm{K}$ Asp1381 further stabilizes the $\alpha \mathrm{J}-\alpha \mathrm{K}$ connection by making a charged interaction with $\alpha \mathrm{J}$ surface exposed Lys1365 and Lys1369 (Fig. 3a, b). Interestingly, the side chain of Arg1359 from the $\alpha$ J helix protrudes from the junction of $\alpha \mathrm{N} 1, \alpha \mathrm{J}$ and $\alpha \mathrm{K}$ such that it makes extensive interactions with $\alpha \mathrm{N} 1$ residues Thr960 and Leu963 as well as $\alpha \mathrm{K}$ Leu1388. The guanidinium group of Arg1359 makes H-bond interactions with the hydroxyl group of $\alpha \mathrm{N} 1$ Thr960 as well as the main chain carbonyl oxygen of $\alpha \mathrm{K}$ Leu1388 (Fig. 3b), thus stabilizing the $\alpha \mathrm{N} 1-\alpha \mathrm{J}-\alpha \mathrm{K}$ junction. Lastly, while the $\alpha \mathrm{L}$ helix interacts with $\alpha \mathrm{I}$ of the PsK domain, this interaction is dominated by backbone, rather than side-chain, interactions. Together, these interactions, combined with the hydrophobic interactions of the Phe1045 (N-lobe), Trp1382 ( $\alpha \mathrm{K})$ and Phe974 $(\alpha \mathrm{N} 1)$ triad (Fig. 2a), contribute to the structural integrity of the $\mathrm{N}$ - and C-regulatory helices and their anchoring to the PsK domain.

Hydrophobic hot spots drive the SgK223 high-affinity dimer. The dimer interface of SgK223 observed in the crystal structure, formed by the unique arrangement of $\alpha \mathrm{N} 1$ and $\alpha$ J into a 'XX'shaped helices bundle, is dominated by hydrophobic interactions. Among these, the most prominent interactions are made through $\alpha \mathrm{N} 1$ Tyr959 and $\alpha \mathrm{J}$ Met1363, whose side chains are buried within the hydrophobic core at the 'XX' interface (Fig. 3c). This intimate packing results in a short-distance $\mathrm{H}$-bond between the hydroxyl groups of Tyr959 from both monomers. The side chain of Met1363 projects from the core to make extensive hydrophobic interactions with $\alpha \mathrm{N} 1$ residues Ser962, Leu963, Leu966 and $\alpha$ J residues Met1364 and Ala1367 (Fig. 3c). Additional hydrophobic interactions at the $\alpha \mathrm{N} 1-\alpha \mathrm{J}$ interface include those made by $\alpha \mathrm{N} 1$ leucines 955 and 966 (Fig. 3d). Interestingly, the side chain of 

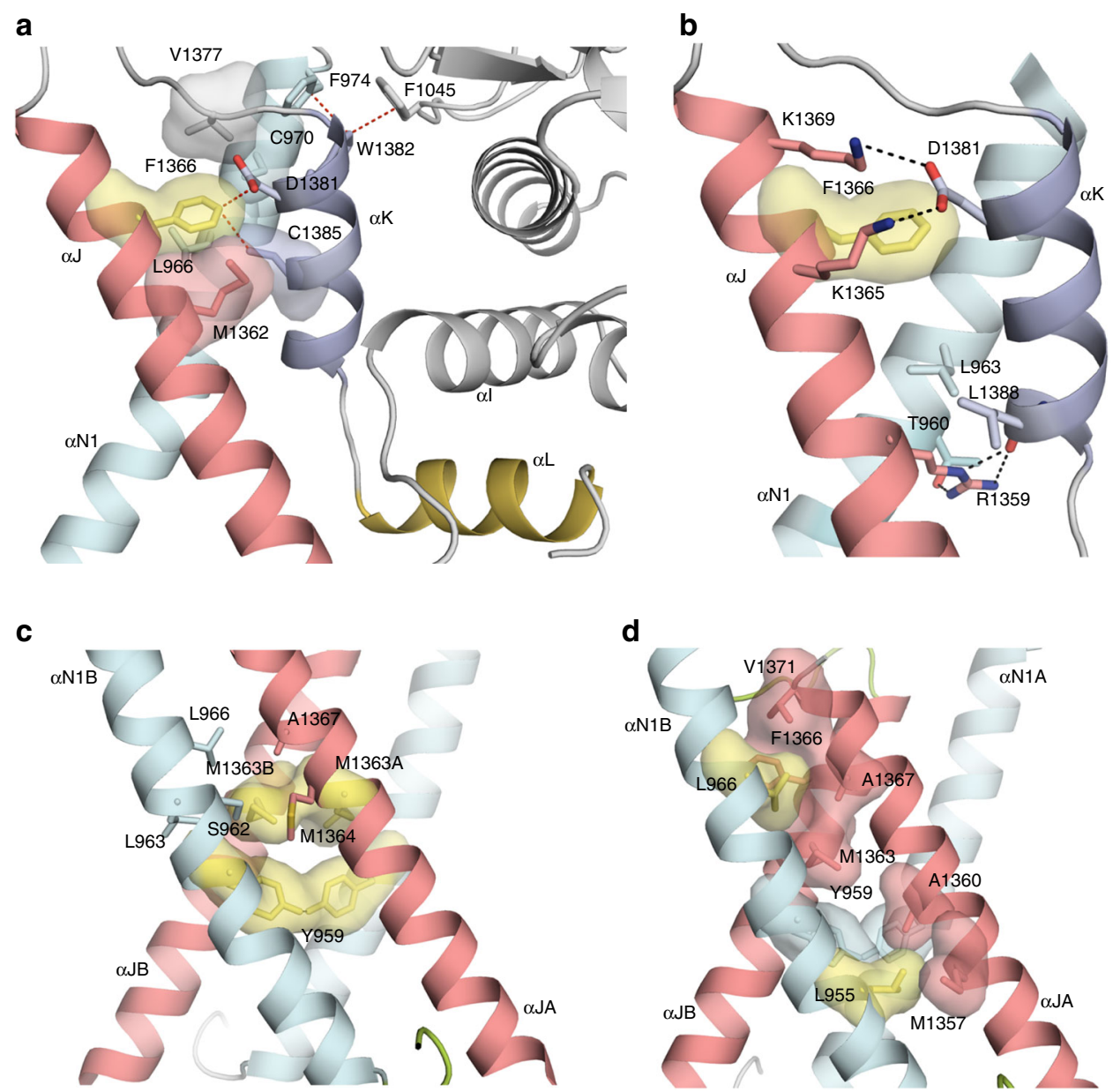

Fig. 3 SgK223 forms a high-affinity dimer. a, b Interaction between $\alpha \mathrm{N} 1, \alpha J$ and $\alpha \mathrm{K}$ helices and the PsK domain. c Met1363 and Tyr959 side chains are buried within the hydrophobic core at the ' $X X$ ' dimer interface between $\alpha \mathrm{N} 1$ and $\alpha$ J helices. $\mathbf{d}$ Hydrophobic interaction surrounding Leu955 and Leu966 at the dimer interface. $\alpha \mathrm{N} 1$, cyan; PK domain, grey; $\alpha$ J, salmon; $\alpha \mathrm{K}$ violet; $\alpha \mathrm{L}$ yellow; h-bond and van der Waals interactions are shown in black and red dashed lines, respectively

Leu966, which occupies the 'knob' position, is protruding into a hydrophobic 'hole' formed by $\alpha$ J residues Met1363, Phe1366, Ala1367 and Val1371. Likewise, hydrophobic residues $\alpha \mathrm{N} 1$ Tyr959 and $\alpha$ J Met1357 and Ala1360 surround the side chain of Leu955, highlighting the importance of leucines as helixstabilizing residues (Fig. 3d).

To determine the energetic hot spots that contribute to SgK223 dimerization and guide functional studies, we conducted exhaustive alanine scanning mutagenesis of residues within the $\alpha \mathrm{N} 1, \alpha \mathrm{J}$ and $\alpha \mathrm{K}$ helices (Supplementary Fig. 3). The mutants were expressed and purified similarly to WT-SgK223- $\alpha$ N1-PsK-Cter and subjected to TSA to assess the impact of the mutations on the stability of the dimer. We used WT-SgK223- $\alpha \mathrm{N} 1-\mathrm{PsK}-\mathrm{Cter}$ that displays a melting temperature $\left(T_{\mathrm{m}}\right)$ of $49^{\circ} \mathrm{C}$ and $\mathrm{SgK} 223-\mathrm{PsK}-$ Cter without the $\alpha \mathrm{N} 1$ helix, which we previously showed to have a significant reduction in thermal stability with a $T_{\mathrm{m}}$ of $44^{\circ} \mathrm{C}^{12}$, to benchmark the impact of individual mutations. Our mutational analysis clearly identified two clusters of hot spot residues that are critical for dimer stability. The first cluster, located at the core of the $\alpha \mathrm{N} 1 / \alpha \mathrm{J}$ dimer interface, consists of residues Leu955, Leu966, Met1363 and Tyr959. Alanine mutation of Leu955 and Leu966 that are central to the core of the $\alpha \mathrm{N} 1$ helix and Met1363 from the $\alpha J$ helix whose side chain protrudes within the hydrophobic core, all lead to a markedly lower $T_{\mathrm{m}}\left(44^{\circ} \mathrm{C}\right)$ compared to WT, indicative of a loss of stability (Fig. 4a). In contrast, alanine mutations of $\alpha \mathrm{N} 1$ Leu958 and $\alpha$ L Leu1361, two residues located further away from the buried interface, do not impact significantly on the thermal stability of the protein (Fig. 4a). Thermal stability analysis of Y959A resulted in a high fluorescent signal at room temperature, suggesting that this mutation leads to drastic increase in exposed hydrophobic patches, a result consistent with the location of Tyr959 at the centre of the hydrophobic core.

The second cluster identified lies between the helices that mediate dimerization and the N-lobe of the PsK domain. Phe1366, in addition to stabilizing Leu966 into a hydrophobic pocket at the dimer interface, forms an important contact at the junction of the $\alpha \mathrm{N} 1, \alpha \mathrm{J}$ and $\alpha \mathrm{K}$ helices within a SgK223 monomer. As expected, F1366A displays a reduced thermal stability (Fig. 4b). Consistent with this, $\alpha \mathrm{K}$ Asp1381, which directly interacts with Phe1366 and also makes charged interaction with $\alpha$ J lysines 1365 and 1369, shows reduced thermal stability when mutated to alanine (Supplementary Fig. 3). Trp1382, which anchors the N-lobe of the PsK domain through its interaction with $\beta 3-\beta 4$ loop Phe1045 and $\alpha$ N1 Phe974, also shows reduced thermal stability when substituted to alanine (Fig. 4b). Surprisingly, F974A substitution had little effect on stability but it is possible that its substitution is compensated by other interactions at this junction. Substitution of $\alpha \mathrm{J}$ Trp1354 to alanine had a detrimental effect on the stability of the protein highlighting its role in connecting the $\alpha \mathrm{J}$ to the PsK C-lobe (Fig. 4b). Similar to Y959A, R1359A substitution resulted in a 
a

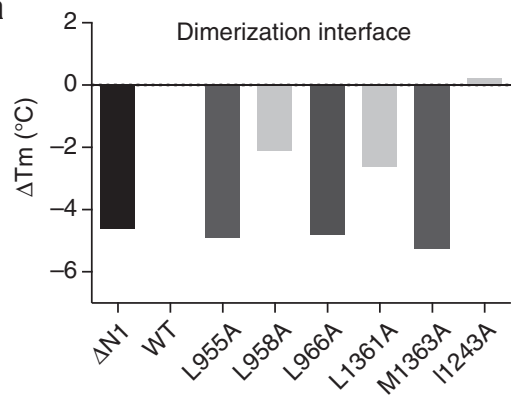

C

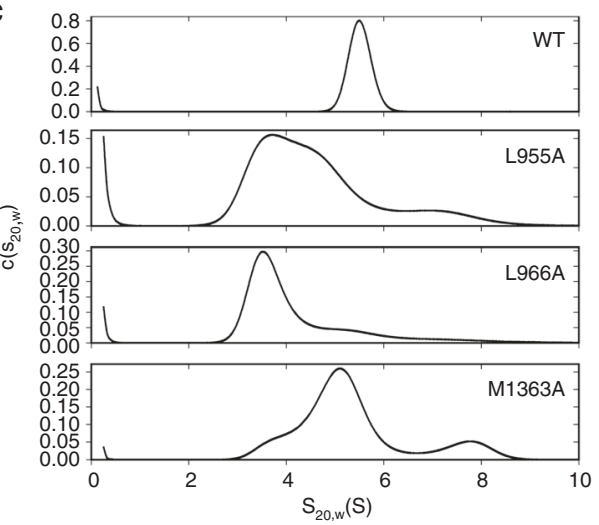

e

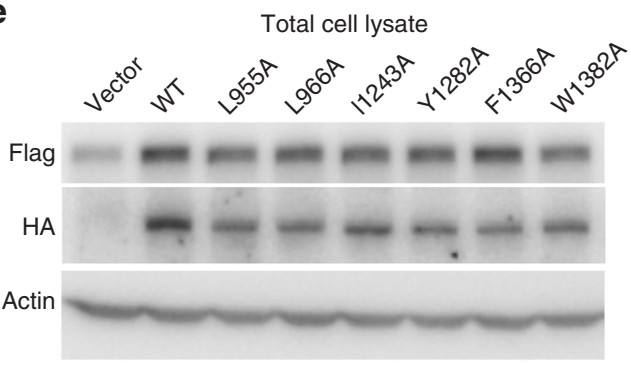

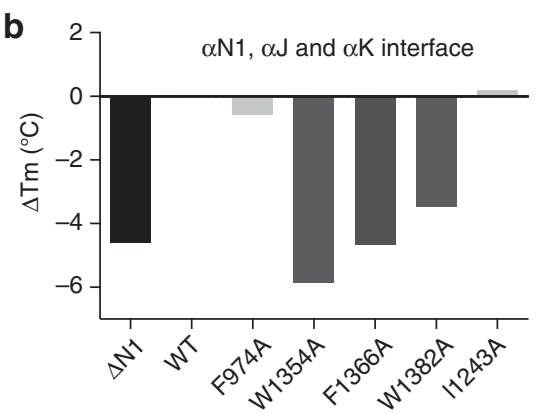

d
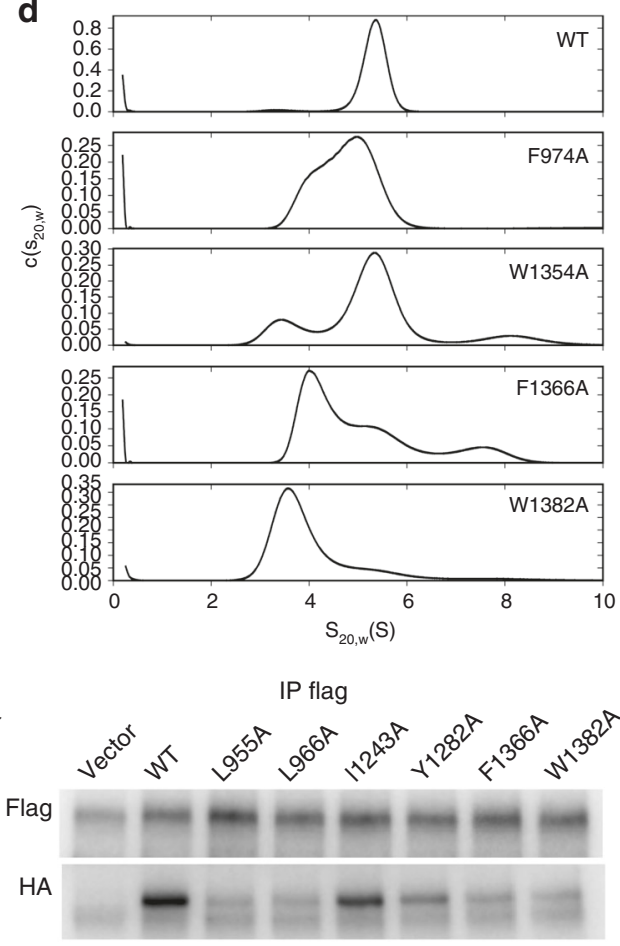

Fig. 4 Two clusters of hydrophobic hot spots mediate SgK223 dimerization. a, b Plots representing the difference in melting temperature between the SgK223- $\alpha$ N1-PsK-Cter (WT), SgK223-PsK-Cter $(\Delta N 1)$ and various mutants within the regulatory helices. $\mathbf{c}, \mathbf{d} c\left(s_{20, w}\right)$ distributions for SgK223- $\alpha$ N1-PsKCter (WT) and mutants, each analysed at a concentration of $3.0 \mu \mathrm{M}$, where the WT protein is primarily dimeric. e Co-immunoprecipitation of Flag-tagged version of SgK223 FL WT with HA-tagged SgK223 FL WT or mutant proteins in HEK293 cells. Lysates were subjected to immunoprecipitation with an antiFLAG antibody followed by Western blotting with anti-FLAG or anti-HA antibodies as indicated. Total cell lysates were also blotted with these antibodies, as well as for actin as a loading control. The data are representative of three independent experiments. See also Supplementary Figs. 3 and 4

high fluorescent signal at room temperature, further highlighting the importance of its side chain protruding internally at the junction of $\alpha \mathrm{N} 1, \alpha J$ and $\alpha \mathrm{K}$ helices and stabilizing critical hydrophobic interactions within a monomer.

To further examine and validate the importance of these two clusters of hot spot residues on dimer formation, we conducted sedimentation velocity experiments at $3.0 \mu \mathrm{M}$, a concentration at which WT SgK223 forms primarily a stable dimer of $\sim 5.6 \mathrm{~s}$ (Fig. $4 \mathrm{c}$ and Supplementary Fig. 3). At this concentration, the two leucine mutants L955A and L966A show significant shifts of the $c$ (s) distribution toward lower sedimentation coefficients with respect to the WT protein, with L966A, displaying a major peak at $\sim 3.5 \mathrm{~s}$, indicating a primarily monomeric state. These data confirm the critical role that these leucines play in maintaining the integrity of the hydrophobic core of the dimer interface (Fig. 4c). Of the $\alpha \mathrm{N} 1, \alpha \mathrm{J}, \alpha \mathrm{K}$ interface mutants, $\alpha \mathrm{K}$ mutant W1382A is primarily monomeric in solution, exhibiting the largest perturbation of dimerization, while $\alpha \mathrm{N} 1$ mutant F974A, despite showing little effect on thermal stability, has a significant impact on dimerization (Fig. $4 \mathrm{~d}$ and Supplementary Fig. 3). This is consistent with the stabilization role that Phe974 and Trp1382 play in anchoring the dimerization domain to the PsK N-lobe (Fig. 2a). As expected, F1366A has a significant impact on dimerization; however, alanine mutation of Trp1354, which connects $\alpha$ J to the PsK C-lobe, displayed a smaller shift towards the monomeric form (Fig. 4d). Of note, a number of these mutants show signals at higher sedimentation coefficients than that observed for WT, suggesting the presence of oligomers larger than dimer perhaps due to native oligomerization or non-specific self-association due to exposure of hydrophobic surfaces.

We next interrogated the contribution of the two hot spots of hydrophobic residues on full-length (FL) SgK223 homotypic association in cells. In HEK293T cells, we co-expressed a Flagtagged version of FL SgK223-WT with FL HA-tagged-WT or 

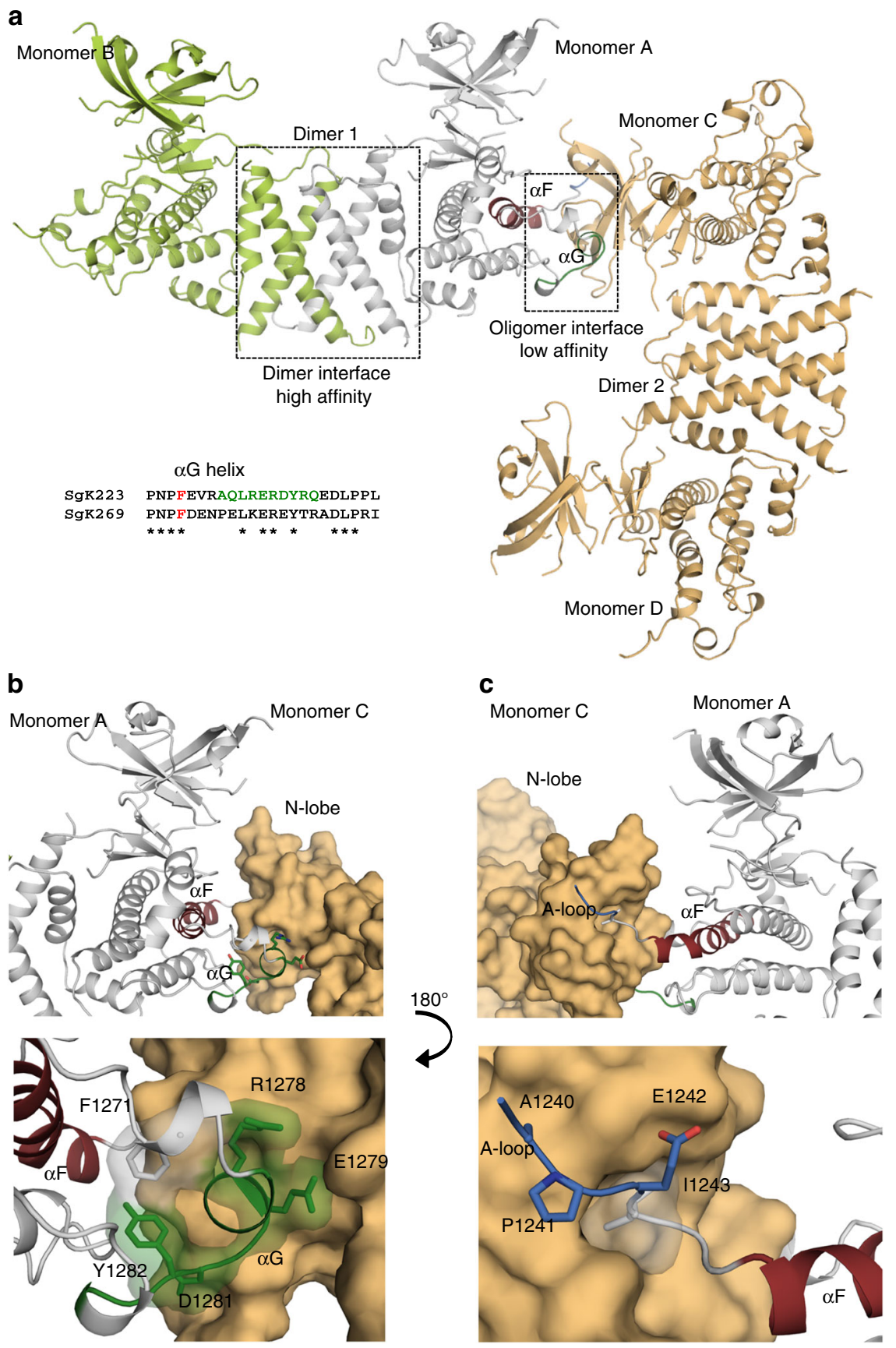

Fig. 5 SgK223 oligomerization. a Identification of the oligomer interface based on crystal packing between two dimers. b Oligomeric interaction made through $\alpha \mathrm{G}$ helix (top) and close up of these interactions (bottom). c Oligomeric interaction made through the end of the activation loop (top) and close up of these interactions (bottom). Monomer B, lime green; Monomer A, grey; Monomer C and D, pale orange; $\alpha G$, green; $\alpha F$, brick red; A-loop, blue. See also Supplementary Fig. 4

mutant proteins characterised as monomeric in our in vitro studies, and studied self-association by co-immunoprecipitation. These experiments revealed that while the differently tagged WT proteins co-immunoprecipitated, as previously described ${ }^{10}$, SgK223 self-association was markedly reduced upon mutations to alanine of the residues Leu955 and Leu966 located at the core of the dimer interface and of the residues Phe 1366 and Trp1382 located at the dimerization-PsK domain junction (Fig. 4e).

In summary, the mutagenesis analysis, combined with coimmunoprecipitation studies clearly identified two clusters of hydrophobic residues that are indispensable for dimer stability in vitro and SgK223 homotypic association in cells. Interestingly, these two hydrophobic hot spots are highly conserved in SgK269 (Supplementary Fig. 3), suggesting that SgK269 will adopt a similar mode of dimerization to that observed for SgK223.

Role of the $\alpha \mathrm{G} / \mathrm{A}$-loop in SgK223 oligomerization. Given that the AUC data showed the presence of higher order SgK223 oligomers at concentrations above $18 \mu \mathrm{M}$ (Supplementary Fig. 1), we investigated which other interfaces could contribute to this oligomerization process by analysing the symmetry related molecules within our crystal packing. Interestingly, analysis through PDBePISA indicated the presence of an interface 
a

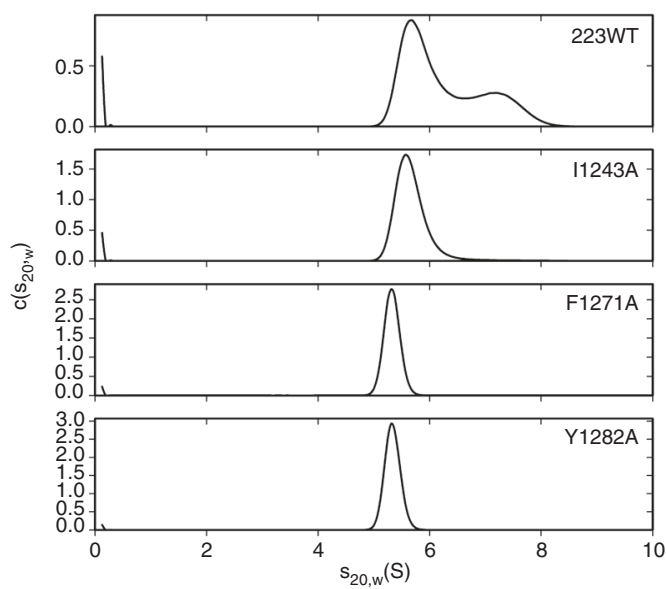

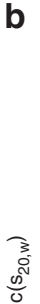

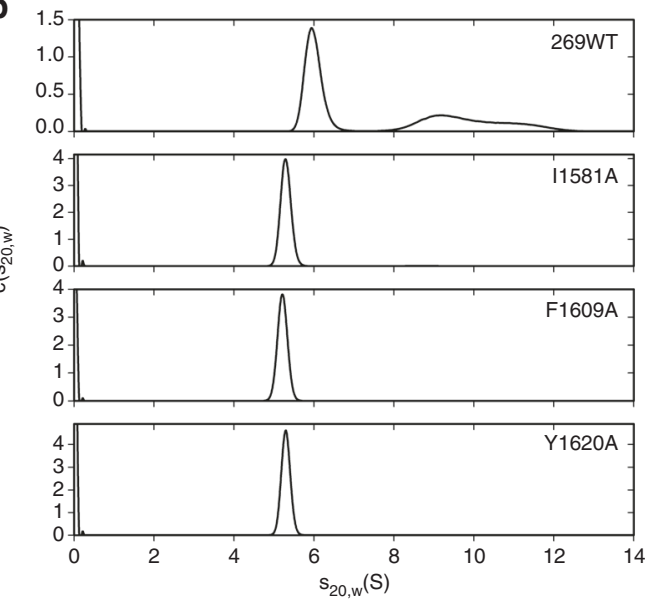

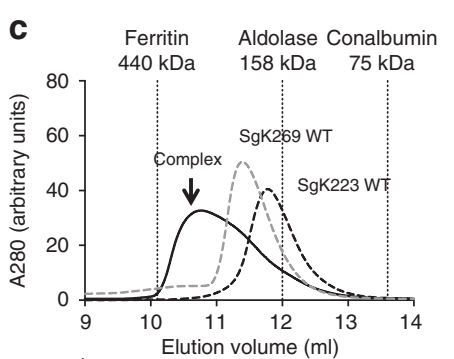
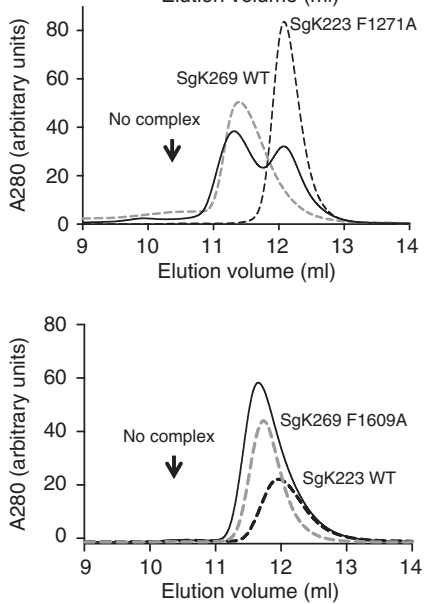

Fig. 6 Characterization of SgK223 and SgK269 oligomerization interface. a c(s $\left.s_{20, w}\right)$ distributions for SgK223- $\alpha$ N1-PsK-Cter (WT) and mutants at the oligomeric interface, each analysed at a concentration of $20.0 \mu \mathrm{M}$, where the WT protein displays significant formation of oligomers larger than dimer. b $c\left(s_{20, w}\right)$ distributions for SgK269- $\alpha$ N1-PsK-Cter $(W T)$ and mutants at the oligomeric interface, each analysed at a concentration of $20.0 \mu \mathrm{M}$, where the WT protein displays significant formation of oligomers larger than dimer. c Size-exclusion chromatography analysis (Superdex $200^{\mathrm{TM}}$ Increase $10 / 300 \mathrm{GL}$, GE Healthcare) of SgK223 and SgK269 complex. Incubation of SgK223- $\alpha$ N1-PsK-Cter (WT) with SgK269- $\alpha$ N1-PsK-Cter (WT) at 1:1 ratio results in a complex formation (top). Incubation of SgK223- $\alpha$ N1-PsK-Cter F1271A mutant with SgK269- $\alpha$ N1-PsK-Cter (WT) does not form a complex (middle). Incubation of SgK269- $\alpha$ N1-PsK-Cter F1609A mutant with SgK223- $\alpha$ N1-PsK-Cter (WT) does not form a complex (bottom). SgK223- $\alpha$ N1-PsK-Cter (WT) and mutants in black dashed lines; SgK269- $\alpha$ N1-PsK-Cter (WT) and mutants in grey dashed lines; complex of SgK223- $\alpha$ N1-PK-Cter and SgK269- $\alpha$ N1-PsKCter in black line. Elution volume of molecular weight standards Ferritin ( $440 \mathrm{kDa})$, Aldolase $(158 \mathrm{kDa})$ and Conalbumin $(75 \mathrm{kDa})$ is shown in vertical dotted lines. See also Supplementary Fig. 5

between the symmetry related dimers, which buries a surface area of $824 \AA^{2}$ (Fig. 5a). Residues from the $\alpha \mathrm{F}$ and $\alpha \mathrm{G}$ helices and the end of the A-loop contribute to this interface by intimately packing into a groove created by N-lobe residues Leu994, Trp989, Tyr1009, Ile1025, Phe1054, Val1058 and Val1089 of the PsK domain (Supplementary Fig. 4). However, only a few residues from the $\alpha \mathrm{G} / \mathrm{A}$-loop site interact directly with the symmetry related dimer (Fig. 5b). The most obvious interaction is through A-loop Ile1243, whose side chain fits snugly into the N-lobe groove to make two main chain H-bonds with Trp989 and Ser1057. In addition, Ile1243, Pro1241 and Glu1242, which mark the end of the 'APE' motif of the A-loop, are involved in various interactions with the $\beta 4-\beta 5$ sheets of the N-lobe of the symmetry related molecule (Fig. 5c; Supplementary Fig. 4).

To establish the role of the $\alpha \mathrm{G}$ helix/A-loop interface in SgK223 homo-oligomerization, we generated alanine mutations of Phe1271, Arg1278, Glu1279, Asp1281, Tyr1282 and Ile1243. Analysis by SEC indicated that $\alpha \mathrm{G}-\mathrm{Ph} 1271$ and -Tyr1282 and A- loop Ile1243 mutants showed a sharper elution profile indicative of a reduced amount of higher order oligomers compared to the WT protein (Supplementary Fig. 4). To confirm the absence of higher order oligomers, we conducted sedimentation velocity experiments at $20 \mu \mathrm{M}$, a concentration at which WT SgK223 shows self-association and the presence of higher order oligomeric species (Fig. 6a and Supplementary Fig. 5). Sedimentation velocity analysis of F1271A and Y1282A showed loss of higher order oligomers and resulted in $c(s)$ distributions similar to the WT protein at a concentration of $3.0 \mu \mathrm{M}$ (c.f. Fig. 4). This indicates that these mutants form stable dimeric structures in solution at raised concentrations. I1243A also showed significant reduction of higher order oligomer formation; however, the $c(s)$ distribution suggests that a small amount of residual oligomerization remains. To confirm that these oligomerization defective mutants did not perturb formation of the core dimer, we conducted SAXS analysis of I1243A (Supplementary Fig. 5). Data collected on I1243A were monodisperse, as indicated by Guinier 
a
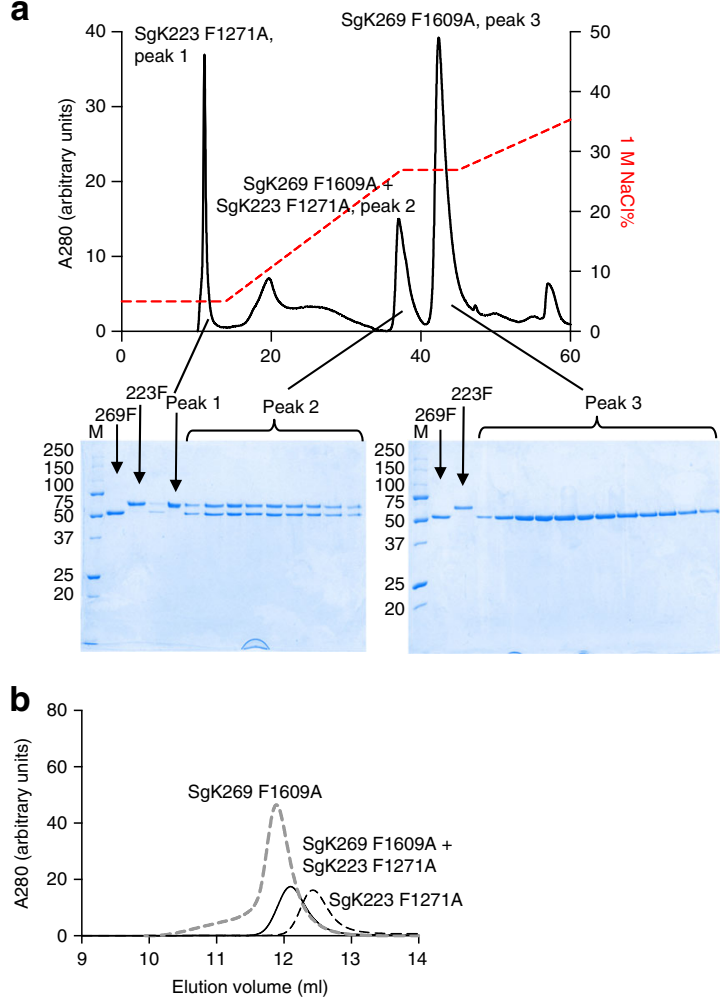

C
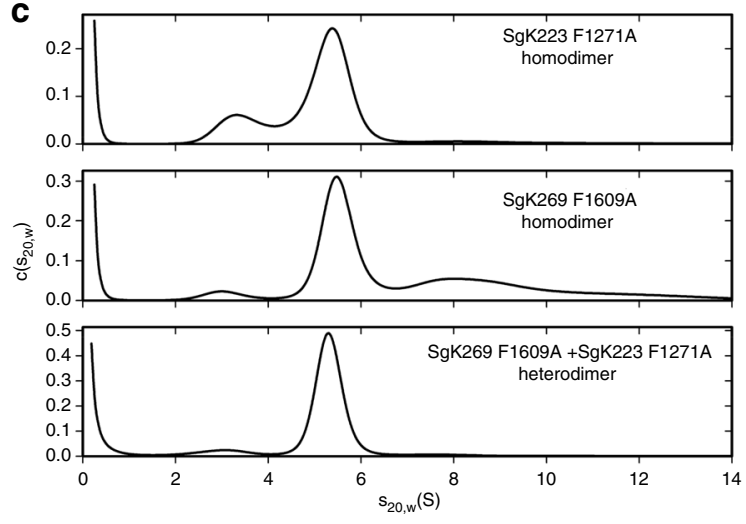

Fig. 7 Heterodimerization of SgK223 and SgK269. a Mono Q (5/50 GL, GE Healthcare) profile of SgK223- $\alpha$ N1-PsK-Cter F1271A/His tag cleaved SgK269- $\alpha$ N1-PsK-Cter F1609A heterodimer complex. SgK223- $\alpha$ N1-PsKCter F1271A and His tag cleaved SgK269- $\alpha$ N1-PsK-Cter F1609A were mixed in equal molar ratio at $20 \mu \mathrm{M}$ and incubated on ice overnight. The resulting complex was run on Mono $Q$ and eluted by applying 5-60\% linear gradient of $1 \mathrm{M} \mathrm{NaCl}$. Protein fractions corresponding to the three main peaks of the homodimer of SgK223- $\alpha$ N1-PsK-Cter F1271A (peak 1), heterodimer of SgK223- $\alpha$ N1-PsK-Cter F1271A and SgK269- $\alpha$ N1-PsK-Cter F1609A (peak 2) and homodimer of SgK269- $\alpha$ N1-PsK-Cter F1609A (peak 3) are shown on an SDS-PAGE along with the molecular weight markers. $\mathbf{b}$ Size exclusion chromatography analysis (Superdex $200^{\mathrm{TM}}$ Increase 10/ 300 GL, GE Healthcare) analysis of SgK223- $\alpha$ N1-PsK-Cter F1271A homodimer shown in black dashed line, SgK223- $\alpha$ N1-PsK-Cter F1271A /SgK269- $\alpha$ N1-PsK-Cter F1609A heterodimer shown in black line and SgK269- $\alpha$ N1-PsK-Cter F1609A homodimer shown in grey dashed line. c $c\left(s_{20, w}\right)$ distributions for SgK223- $\alpha$ N1-PsK-Cter F1271A and SgK269- $\alpha$ N1PsK-Cter F1609A homodimers in comparison to SgK223- $\alpha$ N1-PsK-Cter F1271A and SgK269- $\alpha$ N1-PsK-Cter F1609A heterodimer, each analysed at a concentration of $1.2 \mu \mathrm{M}$. See also Supplementary Fig. 6 analysis, with a maximum particle dimension $\left(D_{\max }\right)$ of $130 \AA$, akin to the WT protein (Fig. 1f), consistent with I1243A existing as a stable dimer in solution.

To further confirm that the oligomerization interface is not a crystal packing artefact, we generated alanine mutants of the corresponding SgK223 $\alpha$ G/A-loop residues in the SgK269- $\alpha \mathrm{N} 1-$ PsK-Cter construct. As per SgK223 mutants, sedimentation velocity experiments of SgK269 $\alpha \mathrm{G}$ (Phe1609, Tyr1620) and A-loop (Ile1581) mutants at $20 \mu \mathrm{M}$, showed loss of higher-order oligomers compared to SgK269-WT (Fig. 6b; Supplementary Fig. 5). These data re-iterate the role that the $\alpha \mathrm{G} / \mathrm{A}$-loop plays in the formation of SgK223 and SgK269 higher-order oligomeric complexes in vitro.

We next sought to characterize the contribution of the $\alpha \mathrm{G} / \mathrm{A}$ loop on FL SgK223 homotypic association in cells. We conducted similar experiments to those described above for the characterization of the dimerization mutants and co-expressed in HEK293T cells a Flag-tagged version of FL SgK223-WT with FL HA-tagged-WT and I1243A or Y1282A mutants. While Aloop I1243A has no detectable impact on self-association, $\alpha G$ Y1282A mutant showed reduced SgK223 self-association (Fig. 4e and Supplementary Fig. 4), indicating that the $\alpha G$ may represent an interface that contributes to SgK223 self-association. However, the impact of the $\alpha \mathrm{G}$ mutations on SgK223 homotypic association is less drastic to that observed when mutating the hot spot residues at the dimerization interface and at the junction between the dimerization domain and the PsK domain (Fig. 4e). This result is in agreement with our SEC and AUC data, which indicate that the oligomerization interface is a low-affinity interface (in the $\mu \mathrm{M}$ range) compared to the high-affinity dimerization interface.

SgK223-SgK269 heterotypic association in vitro. We previously demonstrated, using $\alpha \mathrm{N} 1$ and PsK-Cter deletion constructs, a key role for these regions in mediating SgK223-SgK269 heterotypic association in vitro and in cells, a mechanism that is critical for promoting cell migration of MCF-10A mammary epithelial cells and serves to diversify signalling output ${ }^{10}$. Our detailed structurebased mutational analysis, which clearly identified two clusters of hot spot residues that are critical for dimer stability and highlighted a potential role for the $\alpha \mathrm{G} / \mathrm{A}$-loop in mediating oligomerization, now allowed us to interrogate the relative contribution of each of these three structural elements to SgK223-SgK269 heterotypic association.

We first sought to investigate the oligomerization interface and if the $\alpha \mathrm{G}$ helix F1271A and Y1282A and A-loop I1243A mutants shown to be defective in SgK223 oligomerization in vitro have any impact on heterotypic association of recombinant SgK223and SgK269- $\alpha$ N1-PsK-Cter proteins using SEC analysis. We previously showed using this technique, that pre-incubation of recombinant SgK223-WT ( $\alpha$ N1-PsK-Cter) with SgK269-WT $(\alpha \mathrm{N} 1-\mathrm{PsK}-\mathrm{Cter})$ at a final concentration of $30 \mu \mathrm{M}$ resulted in a marked shift in their elution profiles compared to when each protein was analysed in isolation ${ }^{10}$. Interestingly, this shift towards the formation of a high molecular weight heterocomplex between SgK223 and SgK269 was not observed with any of the three SgK223 mutants that were shown to be impaired in SgK223 self-oligomerization (Fig. 6c and Supplementary Fig. 5). Similarly, no shift towards the formation of a hetero-complex between SgK223 and SgK269 was observed when recombinant SgK223-WT ( $\alpha$ N1-PsK-Cter) was incubated with the SgK269 oligomerization interface mutant $\alpha \mathrm{G} \mathrm{F1609A} \mathrm{(Fig.} \mathrm{6c).}$

These data further demonstrate the importance of the $\alpha \mathrm{G} / \mathrm{A}$ loop in the scaffolding function of SgK223 and SgK269 in vitro. However, it should be noted that the SEC experiments were 
undertaken by mixing preformed SgK223 and SgK269 homodimers, which, given the high affinity of the dimerization interface compared to that of the oligomerization interface, may bias heterotypic association towards the use of the oligomerization interface.

To determine whether SgK223 and SgK269 - $\alpha$ N1-PsK-Cter constructs could hetero-dimerize in vitro through the dimerization interface, we exploited the different overall charge properties of SgK223 and SgK269- $\alpha$ N1-PsK-Cter constructs. We reasoned that the SgK223-SgK269 heterodimer should elute at a different salt concentration from an anion exchange column compared to that of the respective homodimers. We used SgK223 and SgK269$\alpha \mathrm{N} 1$-PsK-Cter $\alpha \mathrm{G}$ mutants to prevent any oligomerization via the $\alpha \mathrm{G}$, while keeping the dimerization interface intact. Incubation of SgK223- $\alpha$ N1-PsK-Cter F1271A with His-tag cleaved SgK269$\alpha \mathrm{N} 1-\mathrm{PsK}-\mathrm{Cter} \mathrm{F} 1609 \mathrm{~A}$ was conducted overnight at an equivalent molar ratio to achieve a final concentration of $20 \mu \mathrm{M}$ prior to loading onto a Mono Q column. These experiments revealed the presence of SgK223-SgK269 heterodimer, eluting at a distinct salt concentration to that of SgK269 homodimer, while His-SgK223$\alpha \mathrm{N} 1-\mathrm{PsK}-\mathrm{Cter}$ homodimer eluted in the flow-through (Fig. 7a). The integrity and molecular weight of the heterodimer purified by Mono Q column, was further confirmed by SEC analysis (Fig. 7b). Sedimentation velocity experiments of the SgK223-SgK269 heterodimer isolated from SEC (Fig. 7b) showed a homogeneous species with a sedimentation coefficient consistent with a dimer (Fig. $7 \mathrm{c}$ and Supplementary Fig. 6). The appearance of signal in the $c\left(s_{20, w}\right)$ distributions at lower or higher sedimentation coefficients than the dimer peak is attributed to partial degradation of the proteins during the long incubation and processing times required.

SgK223-SgK269 heterotypic association in cells. To characterize the contribution of the dimerization and oligomerization interfaces on $\mathrm{SgK} 223-\mathrm{SgK} 269$ heterotypic association in cells, we undertook co-expression studies in SgK223 knock-out (KO) MCF-10A cells in order to remove any potential contribution of endogenous SgK223. The four mutants identified as defective in dimerization (L955A, L966A, F1366A and W1382A) and the two $\alpha \mathrm{G}$ mutants identified as defective in oligomerization (I1243A and Y1282A) in vitro were expressed as HA-tagged versions and association with endogenous SgK269 was assayed by co-immunoprecipitation studies (Fig. 8a). These experiments revealed that while HA-tagged SgK223-WT was able to co-immunoprecipitate endogenous FL SgK269-WT, as previously described $^{10}$, SgK223 heterotypic association with SgK269 was abolished with all four $\mathrm{SgK} 223$ dimerization mutants. These data suggest that SgK223-SgK269 interaction occurs directly via the dimerization interface, a finding consistent with the demonstrated heterodimerization of SgK223 and SgK269 $\alpha$ G mutants in vitro (Fig. 7) and the high level of conservation of the dimerization domain between SgK223 and SgK269 (Supplementary Fig. 3). In contrast, no detectable impact on SgK223-SgK269 heterotypic association was observed with the $\alpha \mathrm{G} / \mathrm{A}$-loop SgK223 mutants (Fig. 8a). However, based on our structural and biochemical data, we cannot exclude a role for the $\alpha \mathrm{G} / \mathrm{N}$-lobe in SgK223-SgK269 interaction. The dimerization defective mutations, located at the core of the $\alpha \mathrm{N} 1 / \alpha \mathrm{J}$ dimer interface and at the junction between the dimerization domain and the N-lobe of the PsK domain, are likely to affect the relative orientation of the N-lobe of the PsK domain, and hence the positioning of the $\alpha \mathrm{G} / \mathrm{N}$-lobe interface, which may impair association with full-length SgK269 in vivo. In contrast, the mutations introduced on the $\alpha \mathrm{G} / \mathrm{A}$-loop would have limited impact on the overall conformation of the PsK domain. Further mutational analysis directed at drastically modifying the
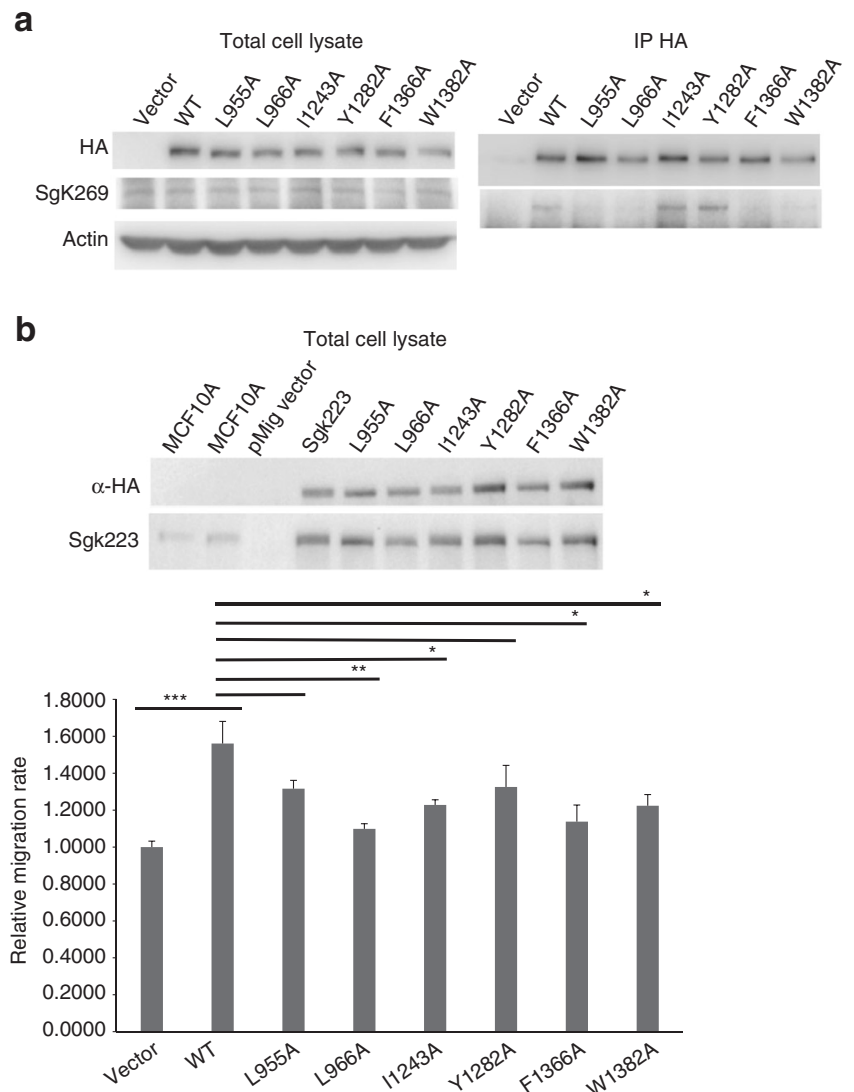

Fig. 8 SgK223-SgK269 heterotypic association in cells and SgK223 function. a Co-immunoprecipitation of exogenous $\mathrm{HA}$-tagged version of $\mathrm{FL}$ WT SgK223 or mutant proteins with endogenous SgK269 in MCF-10A SgK223 KO cells. Lysates were subjected to immunoprecipitation with an anti-HA antibody followed by western blotting with anti-HA or anti-SgK269 antibodies as indicated. Total cell lysates were also blotted with these antibodies, as well as for actin as a loading control. The data are representative of two independent experiments. $\mathbf{b}$ Effect of interface mutations on SgK223-promoted cell migration. Western blotting of total cell lysates to confirm expression levels of SgK223 FL WT and mutants in MCF-10A parental and SgK223 KO cells. Lysates were blotted with the indicated antibodies (top). Effect of SgK223 FL WT and mutants on cell migration in MCF-10A SgK223 KO cells (bottom). Relative wound closure was measured using TScratch software ${ }^{59}$ and used to calculate the cell migration rate. This is expressed relative to vector control, which was arbitrarily set at 1.0. Data represent mean \pm SEM from four independent experiments. ${ }^{\star \star \star} p<0.005$ (for comparison of WT to vector), ${ }^{\star} p<0.05$, ${ }^{\star \star} p<0.01$ (for comparison of mutants to WT). See also Supplementary Fig. 7

$\alpha \mathrm{G} / \mathrm{A}$-loop conformation and targeting the complementary PsK $\mathrm{N}$-lobe interface will be necessary to fully interrogate the contribution of the low-affinity $\alpha \mathrm{G} / \mathrm{A}-\mathrm{loop} / \mathrm{N}$-lobe interface, independently from the high affinity dimerization interface, on SgK223-SgK269 heterotypic association in cells.

In order to validate the impact of the dimerization and oligomerization mutations on SgK223 biological function, we utilized cell migration assays, as overexpression of SgK223 enhances cell migration ${ }^{19}$ and SgK223 is required for SgK269 to promote cell motility in MCF-10A cells ${ }^{10}$. Introduction of SgK223-WT or specific mutants into SgK223 KO MCF-10A cells resulted in modest overexpression of the proteins at equivalent levels (Fig. 8b and Supplementary Fig. 7). Expression of SgK223WT resulted in an $\sim 1.5$-fold enhancement of cell migration rate relative to the vector control. However, the ability of SgK223 to 
enhance migration was significantly reduced for L966A (dimerization interface), F1366A and W1382A (junction between the dimerization domains and the PsK domain) and I1243A (A-loop). A non-significant trend for decreased migration was observed for dimerization mutant L955A and $\alpha \mathrm{G}$ helix mutant Y1282A (Fig. 8b). Overall, these results provide strong evidence that the integrity of both the dimerization and oligomerization interfaces is required for optimal promotion of cell migration by SgK223.

\section{Discussion}

In this study, we used X-ray crystallography to provide highresolution insights into the molecular mechanism that drives SgK223 self-association. Our structure is a remarkable example of how pseudokinases have compromised their nucleotide binding function to evolve as dynamic scaffolds. Despite an overall common kinase fold, the pseudokinase domain of SgK223 displays striking structural differences in its N-lobe when compared to conventional active kinases. SgK223 PsK domain is characterized by an inaccessible ATP-binding site, a structural feature conserved with the previously characterized pseudokinases VRK3, TRB1, ROP2 and ROR2 $9,13,26,27$. The occluded ATPbinding site in SgK223 PsK domain is a result of a conformational repositioning of the $\mathrm{N}$-lobe in relation to the $\mathrm{C}$-lobe, driven by a non-canonical interaction between the conserved Lys from the $\beta 3$-strand and Gln1048 from the loop preceding the $\beta 4$ strand, an interaction that is further stabilized by the unique Phe1045 (Nlobe), Trp1382 $(\alpha \mathrm{K})$ and Phe974 ( $\alpha \mathrm{N} 1)$ triad (Fig. 2a). Without geometrical constraints required for catalytic activity, the SgK223 pseudoactive site seems to have evolved new interactions to accommodate the positioning of the adjacent structural elements.

Pseudokinase domains, like kinase domains, are often surrounded by accessory regions that provide additional, often regulatory functionalities. However, many aspects of the noncatalytic signalling functions of pseudokinases remain poorly understood. Deciphering the structural diversity of these noncatalytic elements is critical to understand how these catalytically inert modules disseminate or coordinate signal outputs. Recent work on the pseudokinase Mixed Lineage Kinase domain-like (MLKL), a key effector of necroptosis involved in innate immunity and inflammation, elegantly demonstrates how a conformational change of the pseudokinase domain unleashes the $\mathrm{N}$-terminal four-helix bundle domain, subsequently leading to MLKL oligomerization, membrane translocation and permeabilization $^{7,28}$. Structural studies of the PAN3 adaptor pseudokinase clearly highlights how a coiled-coil region and a helix directly surrounding the PsK domain, drive an asymmetric domain swapped dimer that creates a docking site for the deadenylase PAN2 to form a catalytically competent PAN2-PAN3 deadenylase complex that regulates mRNA degradation ${ }^{29,30}$. The crystal structure of $\mathrm{SgK} 223$ presented here is another remarkable example of the structural and functional diversity of the pseudokinase family. Our structure reveals how the $\alpha \mathrm{N} 1$ helix $\mathrm{N}$-terminal to the pseudokinase domain packs against helices in the C-terminal domain in cis to form a unique ' $\mathrm{X}$ '-shaped helix bundle, which also nucleates the assembly of a high affinity ' $\mathrm{XX}$ ' dimer. By performing extensive mutagenesis in conjunction with TSA and AUC, we identified two hydrophobic clusters of hot spot residues that contribute to the formation of a high affinity dimer. The strict conservation of these residues in SgK269 (Supplementary Fig. 3) indicates that this dimer packing arrangement is likely to be conserved in SgK269. This unique signal-independent homotypic dimerization, not previously reported in any kinase or pseudokinase structures, is likely to play an important role in promoting the structural rigidity necessary to drive SgK223's scaffolding function, where the two central PEST regions bring the effectors into close proximity to facilitate their transactivation, in a manner analogous to the cytoplasmic tails of dimerized receptor tyrosine kinases (Supplementary Fig. 8). In support of this model, the SgK223-Csk interaction stimulates Csk kinase activity, most likely through a trans-activation event ${ }^{18}$.

Considering that SgK223 and SgK269 have a distinct repertoire of known effectors, homotypic or heterotypic association is likely to provide a means to regulate signalling complex assembly and signal output ${ }^{10}$. The structural studies presented herein have enabled the identification of the key molecular interfaces that underpin SgK223 self-association and may be critical for SgK223-SgK269 heterotypic association. It is tempting to speculate that the specific dimer arrangement would allow the $\alpha \mathrm{G}$ helix, which largely bulges out to solvent, to act as a docking site for the assembly of oligomeric complexes. Several studies support a role of the $\alpha \mathrm{G}$ helix/C-terminal A-loop in mediating selective interactions between kinase/pseudokinase domains and substrates/regulators. A classic example of this mode of interaction is the kinase-regulator structure PKA-RI $\alpha^{31}$. This non-catalytic protein interaction function driven by the $\alpha G$ helix is also conserved within the pseudokinase family. The pseudokinase integrin linked kinase (ILK) interacts with its activator $\alpha$-parvin $\mathrm{CH} 2$ for focal adhesion targeting through a protruding surface on the Clobe that comprises the $\alpha E F$ and $\alpha G$ helices and the $C$ terminus of the activation loop (Supplementary Fig. 8). Mutations of critical residues driving this interaction completely abolished the binding of parvin to ILK in vivo and dramatically impaired ILK localization to focal adhesion sites ${ }^{11}$

A key issue arising from our studies is the relative contribution of the dimerization and oligomerization interfaces to SgK223/ SgK269 homo- and heterotypic association. In the case of SgK223, our data support a contribution from both interfaces for homotypic association in vitro and in vivo. Mutations in either interface negatively impacted the ability of SgK223 to promote cell migration, indicating that dimerization and formation of higher order oligomers is required for biological activity. However, while we could demonstrate a role for both interfaces in SgK223/SgK269 heterotypic association in vitro, and a role for the dimerization interface in heterotypic association in vivo, our coIP data do not reveal a role for the oligomerization interface in heterotypic association between SgK223 and SgK269 in cells. This indicates that the contribution of the SgK223 oligomerization interface to biological activity is likely reflecting SgK223 homotypic association.

In conclusion, the pseudokinase SgK223 structure presented in this study reveals a novel dimerization domain fold and highlights the remarkable adaptability of the kinase scaffold to accommodate accessory regulatory domains in order to drive noncatalytic functions. The structural identification of the key molecular interfaces that underpin SgK223/SgK269 homo- and hetero-typic association represents a major advance in our fundamental understanding of how pseudokinases function in cell signalling.

\section{Methods}

Recombinant protein expression and purification. Synthetic genes of human SgK223 $\alpha$ N1-PsK-Cter (residues 932-1406), SgK269 $\alpha$ N1-PsK-Cter (residues 1267-1746), truncated SgK223 $\alpha \mathrm{N} 1$ constructs (residues 975-1406) and mutants (GenScript) were cloned into a modified pCOLD IV vector (Takara) that contains a $\mathrm{N}$-terminal His TEV cleavage tag. Recombinant plasmid were used to transform $E$. coli C41(DE3) (Supplementary Table 1). Protein expression was carried out in $E$. coli $\mathrm{C} 41(\mathrm{DE} 3)$ strain and expression cultures were grown according to the autoinduction method ${ }^{32}$. For SgK223 purification, cells were resuspended in lysis buffer containing $20 \mathrm{mM}$ Tris $\mathrm{pH} 8.5,500 \mathrm{mM} \mathrm{NaCl}, 10 \% \mathrm{v} / \mathrm{v}$ glycerol, $5 \mathrm{mM}$ imidazole, $0.1 \%$ Thesit, $2 \mathrm{mM}$ EDTA and $10 \mathrm{mM}$ DTT, supplemented with Complete EDTAfree Protease Inhibitor Cocktail (Roche) and lysed by sonication. The supernatant was clarified by centrifugation at $45,000 \times g, 4^{\circ} \mathrm{C}, 30 \mathrm{~min}$, filtered and loaded onto a 
$1 \mathrm{ml} \mathrm{Ni}$-NTA resin (Roche). After extensive washes with wash buffer $(20 \mathrm{mM}$ Tris $\mathrm{pH} 8.5,500 \mathrm{M} \mathrm{NaCl}, 10 \% \mathrm{v} / \mathrm{v}$ glycerol, $5 \mathrm{mM}$ imidazole, $2 \mathrm{mM}$ EDTA and $10 \mathrm{mM}$ DTT), the protein was eluted in the wash buffer supplemented with $150 \mathrm{mM}$ imidazole. SgK223 containing fractions were subjected to size exclusion chromatography (SEC) (Superdex-200 16/600, GE Healthcare) in SEC buffer (20 mM Tris $\mathrm{pH} 8.5,200 \mathrm{mM} \mathrm{NaCl}, 5 \% \mathrm{v} / \mathrm{v}$ glycerol, $1 \mathrm{mM}$ TCEP). SgK269 and mutants were purified using a similar protocol as SgK223 except that the $\mathrm{pH}$ of Tris was changed to $\mathrm{pH} 8.0$ during purification and analysis. Fractions containing SgK223 or SgK269 proteins were pooled, concentrated and flash-frozen for storage at $-80^{\circ} \mathrm{C}$. All mutant proteins were subjected to the same purification procedure. The selenomethionine labelled SgK223 was expressed in E. coli C3022I strain (NEB) grown in auto-induction medium in the presence of $125 \mu \mathrm{g} \mathrm{ml}^{-1}$ seleno-methionine ${ }^{32,33}$. The purification of seleno-methionine-labelled SgK223 was similar to the native protein.

Structure determination. Crystals of $\mathrm{SgK} 223$ and of the seleno-methionine substituted SgK223 were obtained by the vapour diffusion method by mixing $150 \mu \mathrm{l}$ of protein at $5 \mathrm{mg} \mathrm{ml}^{-1}$ with $150 \mu$ l of reservoir solution containing $1.6 \mathrm{M} \mathrm{NaH}_{2} \mathrm{PO}_{4} /$ $\mathrm{KH}_{2} \mathrm{PO}_{4}$ and $0.1 \mathrm{M}$ Hepes $\mathrm{pH}$ 7.5. Crystals were flash-frozen in liquid nitrogen in the reservoir solution supplemented with $10 \%$ glycerol. X-ray diffraction data for both native SgK223 and SeMet-SgK223 were collected on the MX2 beamline at the Australian Synchrotron. The native crystal diffracted to $2.95 \AA$ resolution. The data were collected at a wavelength of $0.9537 \AA$ using one crystal and a total rotation of $200^{\circ}$ with a $1^{\circ}$ oscillation range. MAD scan was performed on the SeMet crystal around the selenium edge. The SeMet data sets were collected at wavelength 0.9537 $\AA$ (high energy remote) and $0.9796 \AA$ (inflection point) to resolution between 3.4 and $3.5 \AA$. At each wavelength, two sets of data were collected at two different positions of the crystal in order to minimize radiation damage and improve anomalous and dispersive signal. All diffraction data were processed using $\mathrm{XDS}^{34}$ in primitive tetragonal space group. The SeMet data sets collected at each wavelength were merged and scaled using XSCALE ${ }^{35}$. Table 1 summarizes the datacollection statistics.

Automated experimental phasing were carried out using two-wavelength multiple anomalous diffraction (2W-MAD) phasing protocol of Auto-Rickshaw ${ }^{36}$ The input diffraction data were prepared and converted for use in Auto-Rickshaw using programs of the CCP4 suite ${ }^{37}$. FA values were calculated using the program SHELXC. The maximum resolution for substructure determination and initial phase calculation was set to $4.3 \AA$. All of the seven heavy atoms requested were found using the program SHELXD ${ }^{38}$ and the absolute configuration (hand) of the substructure was determined using the program $\mathrm{ABS}^{39}$. The correct space group was identified to $\mathrm{P}_{3} 2_{1} 2$. The occupancy of all substructure atoms was refined and initial phases were calculated using the program $B P 3^{40}$. Density modification and phase extension were performed to $3.4 \AA$ resolution using RESOLVE ${ }^{41}$. A partial model (337 residues out of the total number of 450 residues) was produced using BUCCANEER $^{42}$. Further the native data and the partial model were used in MRSAD protocol of Auto-Rickshaw ${ }^{43}$ for phase enhancement and model completion. The resulting model contained $87 \%$ of the total number of residues. The model was further improved using manual model building in $\mathrm{COOT}^{44}$, with refinement against the native data set at $2.95 \AA$ using PHENIX ${ }^{45}$. The majority of the residues were visible in the structure with the exception of residues present in surface exposed loops: 932-943, 977-983, 1030-1039, 1065-1081, 1159-1207, 1218-1238 and 1336-1345. The final refined model had 97\% residues in the favoured region and 3\% residues in the allowed region of the Ramachandran plot. The overall structure was validated using MOLPROBITY ${ }^{46}$. All molecular graphics representations were created using PyMOL (The PyMOL Molecular Graphics System, Version 1.7.4.0, Schrödinger, LLC). PDBePISA was used for buried surface area calculation ${ }^{47}$. Sequence alignment was done using Clustal Omega ${ }^{48,49}$.

Thermal shift assay. TSAs were performed as described previously $7,9,12,50$, using a Rotor-Gene Q PCR. The proteins were diluted in $20 \mathrm{mM}$ Tris pH 8.0, $150 \mathrm{mM}$ $\mathrm{NaCl}, 1 \mathrm{mM}$ DTT to $5.3 \mu \mathrm{M}$ final concentration and assayed in a total reaction volume of $25 \mu$ l. SYPRO Orange (Molecular Probes, CA) was used as a fluorescence probe and detected at $530 \mathrm{~nm}$. We used WT-SgK223- $\alpha \mathrm{N} 1-\mathrm{PsK}-\mathrm{Cter}$ that displays a melting temperature $\left(T_{\mathrm{m}}\right)$ of $49^{\circ} \mathrm{C}$ and SgK223-PsK-Cter without $\alpha \mathrm{N} 1$ helix, which we previously showed to have a significant reduction in thermal stability with a $T_{\mathrm{m}}$ of $44^{\circ} \mathrm{C}^{12}$. We also included as control an activation loop mutant (I1243A) located away from the dimerization interface. This mutation had no effect on stability, consistent with its location on the opposite side of the PsK domain.

Analytical size exclusion chromatography. All SEC analyses were performed on a Superdex ${ }^{\mathrm{TM}} 200$ Increase $10 / 300 \mathrm{GL}$ (GE Healthcare) equilibrated in $20 \mathrm{mM}$ Tris $\mathrm{pH} 8.5,200 \mathrm{mM} \mathrm{NaCl}, 5 \% \mathrm{v} / \mathrm{v}$ glycerol, $1 \mathrm{mM}$ TCEP. For single elution analysis, WT proteins and mutants were prepared at the final concentration of $2.79 \mathrm{mg} \mathrm{ml}^{-1}$ $(51.6 \mu \mathrm{M})$ in a total volume of $110 \mu \mathrm{l}$. For complex interaction studies, SgK223 and SgK269 proteins were mixed in equimolar quantity at $29.06 \mu \mathrm{M}$ final concentration in a total reaction volume of $110 \mu \mathrm{l}$, left $15 \mathrm{~min}$ on ice, centrifuged $\left(15,800 \times g, 4^{\circ} \mathrm{C}\right.$, $10 \mathrm{~min}$ ) before loaded onto the column. Protein fractions were analysed by SDSPAGE.
For heterodimerization studies, SgK223- $\alpha$ N1-PsK-Cter Phe1271Ala and His tag cleaved SgK269- $\alpha$ N1-PsK-Cter Phe1609Ala were mixed in equimolar ratio at 20 $\mu \mathrm{M}$ in a total reaction volume of $1 \mathrm{ml}$ and incubated overnight on ice. The complex was then diluted five times in $20 \mathrm{mM}$ Tris $\mathrm{pH} 8.5,50 \mathrm{mM} \mathrm{NaCl} 5 \%$ glycerol, $1 \mathrm{mM}$ TCEP (buffer A) before loading onto a MonoQ (5/50 GL, GE Healthcare) column. SgK223- $\alpha$ N1-PsK-Cter Phe1271Ala and SgK269- $\alpha$ N1-PsK-Cter Phe1609Ala homodimers were separated from heterodimer of SgK223- $\alpha$ N1-PsK-Cter Phe1271Ala and His tag cleaved SgK269- $\alpha$ N1-PsK-Cter Phe1609Ala by applying a linear salt gradient over 60 column volumes starting at $5 \%$ of buffer B $(20 \mathrm{mM}$ Tris pH 8.5, $50 \mathrm{mM} \mathrm{NaCl} 5 \%$ glycerol, $1 \mathrm{mM} \mathrm{TCEP}, 1 \mathrm{M} \mathrm{NaCl}$ ) until $60 \% \mathrm{~B}$. The homodimers and heterodimers peaks were analysed by SDS-PAGE and loaded onto a Superdex ${ }^{\mathrm{TM}} 200$ Increase 10/300 GL (GE Healthcare) equilibrated in $20 \mathrm{mM}$ Tris $\mathrm{pH} 8.5,200 \mathrm{mM} \mathrm{NaCl}, 1 \mathrm{mM}$ TCEP for analysis before being subjected to analytical ultracentrifugation.

Analytical ultracentrifugation. Sedimentation velocity experiments were performed using an XL-I analytical ultracentrifuge (Beckman Coulter) equipped with UV/Vis scanning optics. Buffer reference (20 mM Tris-HCl, $150 \mathrm{mM} \mathrm{NaCl}, \mathrm{pH} 8.0)$ and sample solutions were loaded into $12 \mathrm{~mm}$ double-sector cells with quartz windows and the cells were mounted in an An-60Ti 4-hole rotor. All experiments were conducted at $50,000 \mathrm{rpm}(201,600 \times g)$ and $20^{\circ} \mathrm{C}$, and radial absorbance data were collected at appropriate wavelengths in continuous mode. Data were fitted to a continuous sedimentation coefficient distribution $[c(s)]$ model and converted to standardized sedimentation coefficient $\left(s_{20, \mathrm{w}}\right)$ distributions using SEDFIT ${ }^{51}$. The protein partial specific volumes, buffer density and buffer viscosity were calculated using SEDNTERP ${ }^{52}$. Weight average sedimentation coefficients were determined by integration of $c(s)$ distributions between 2 and $10 \mathrm{~s}$. Molecular weights were calculated from weight average sedimentation coefficients in SEDFIT using the frictional ratio obtained from the fit to the sedimentation velocity data.

Small-angle X-ray scattering. SAXS data collection was performed at the Australian Synchrotron SAXS/WAXS beamline using an inline gel filtration chromatography setup, as described previously ${ }^{7}, 50,53,54$ with the co-flow modification ${ }^{55}$. Summary statistics for data collection and analysis are reported in Supplementary Table 1. A volume of $90 \mu \mathrm{l}$ of purified recombinant SgK223 $\alpha$ N1-PsK-Cter WT at $2.2 \mathrm{mg} \mathrm{ml}^{-1}$ and SgK223 $\alpha \mathrm{N} 1$-PsK-Cter I1243A mutant at $3.5 \mathrm{mg} \mathrm{ml}^{-1}$ were injected onto an inline Superdex $2005 / 150$ column (GE Healthcare) and eluted at a flow rate of $0.2 \mathrm{ml} \mathrm{min}^{-1}$ via a $1.5 \mathrm{~mm}$ glass capillary positioned in the X-ray beam in $20 \mathrm{mM}$ Tris pH 8,5, $200 \mathrm{mM} \mathrm{NaCl}, 1 \mathrm{mM}$ TCEP, $5 \%$ Glycerol at $12^{\circ} \mathrm{C}$. Scattering data were collected on the monodisperse peak (corresponding to the dimeric form) in $2 \mathrm{~s}$ exposures over the course of the elution and $2 \mathrm{D}$ intensity plots with consistent scatter intensities from the peak of the sizing exclusion chromatography run (a total of four WT or seven I1243A patterns) were radially averaged, normalized to sample transmission, with background subtraction performed using the Scatterbrain software (Stephen Mudie, Australian Synchrotron). Guinier analysis of data was performed using PRIMUS ${ }^{56}$ and indirect Fourier transform with GNOM $^{57}$ to obtain the distance distribution function, $P(r)$, and the maximum dimension, $D_{\max }$, of the scattering particle. CRYSOL ${ }^{58}$ was used to calculate theoretical scattering curves from crystal structure atomic coordinates and compare with experimental scattering curves. Comparison of the experimental scatter pattern obtained for SgK223 $\alpha$ N1-PsK-Cter I1243A mutant with the theoretical scatter calculated using the SgK223 $\alpha$ N1-PsK-Cter WT crystal structure coordinates showed concordance $(\chi=0.984)$, consistent with SgK223 $\alpha$ N1-PsK-Cter I1243A existing as a stable dimer in solution and adopting a conformation akin to SgK223 $\alpha$ N1-PsK-Cter-WT

Cell culture and co-immunoprecipitation experiments. HEK 293 and PlatE cells were maintained in Dulbecco's modified Eagle's medium (Gibco) supplemented with $10 \%$ FCS (Serana). MCF-10A SgK223 KO cells were as previously described ${ }^{10}$ and were maintained in Dulbecco's modified Eagle's medium/nutrient mixture F-12 (Invitrogen) supplemented with 5\% (v/v) horse serum (Invitrogen), $20 \mathrm{ng} \mathrm{ml}^{-1}$ human recombinant EGF (R\&D Systems), $0.5 \mu \mathrm{g} \mathrm{ml}^{-1}$ hydrocortisone (Sigma), $100 \mathrm{ng} \mathrm{ml}^{-1}$ cholera toxin (Sigma), $10 \mu \mathrm{g} \mathrm{ml}^{-1}$ bovine insulin (Sigma), 50 units per ml penicillin $\mathrm{G}$ (Invitrogen) and $50 \mu \mathrm{g} \mathrm{ml}^{-1}$ streptomycin sulfate (Invitrogen). Retroviral infection of these cells, transfection of HEK293 cells, and co-immunoprecipitation studies, were also undertaken as previously reported ${ }^{10}$ Western blot analysis were performed using the following antibody dilutions: Flag $(1: 1,000)$, HA (1:1,000), Actin (1:5,000), SgK223 (1:500), SgK269 (1:500) (Supplementary Table 1). Raw uncropped western blot scans are in Supplementary Figs. 4 and 7.

Wound healing assay. MCF-10A SgK223 KO cells expressing wild-type SgK223 and mutants were seeded in 24 -well plates at $2 \times 10^{5}$ cells per well. After $24 \mathrm{~h}$, wounds were made by scraping with a p20 plastic pipette tip across the cell monolayer, and then the cells were cultured with $1 \mu \mathrm{g} \mathrm{ml}^{-1}$ mitomycin $\mathrm{C}$ to prevent cell division. Pictures were taken automatically every $0.5 \mathrm{~h}$ from 0 to $18 \mathrm{~h}$ and from four or five different places in the same well using a Leica AF6000LX Live cell microscope. The percentage of wound area at the different time points was determined using TScratch software ${ }^{59}$ and relative cell migration rate was 
calculated from the slope of plotting the percentage of wound area to time as described ${ }^{60}$. Student's $t$-test was used to calculate $p$ values.

Data availability. The structure of SgK223 $\alpha$ N1-PsK-Cter has been deposited in the Protein Data Bank under ID code PDB:5VE6. All other data supporting the findings of this study are available within the paper, and its Supplementary Information files and are also available from the corresponding authors upon request.

Received: 6 May 2017 Accepted: 1 September 2017

Published online: 27 October 2017

\section{References}

1. Jura, N., Shan, Y., Cao, X., Shaw, D. E. \& Kuriyan, J. Structural analysis of the catalytically inactive kinase domain of the human EGF receptor 3. Proc. Natl Acad. Sci. USA 106, 21608-21613 (2009).

2. Littlefield, P. et al. Structural analysis of the EGFR/HER3 heterodimer reveals the molecular basis for activating HER3 mutations. Sci. Signal. 7, ra114 (2014).

3. Babon, J. J., Lucet, I. S., Murphy, J. M., Nicola, N. A. \& Varghese, L. N. The molecular regulation of Janus kinase (JAK) activation. Biochem. J. 462, 1-13 (2014).

4. Hammaren, H. M. et al. ATP binding to the pseudokinase domain of JAK2 is critical for pathogenic activation. Proc. Natl Acad. Sci. USA 112, 4642-4647 (2015).

5. Lupardus, P. J. et al. Structure of the pseudokinase-kinase domains from protein kinase TYK2 reveals a mechanism for Janus kinase (JAK) autoinhibition. Proc. Natl Acad. Sci. USA 111, 8025-8030 (2014).

6. Zeqiraj, E., Filippi, B. M., Deak, M., Alessi, D. R. \& van Aalten, D. M. Structure of the LKB1-STRAD-MO25 complex reveals an allosteric mechanism of kinase activation. Science 326, 1707-1711 (2009).

7. Murphy, J. M. et al. The pseudokinase MLKL mediates necroptosis via a molecular switch mechanism. Immunity 39, 443-453 (2013).

8. Langeberg, L. K. \& Scott, J. D. Signalling scaffolds and local organization of cellular behaviour. Nat. Rev. Mol. Cell Biol. 16, 232-244 (2015).

9. Murphy, J. M. et al. Molecular mechanism of CCAAT-enhancer binding protein recruitment by the TRIB1 pseudokinase. Structure 23, 2111-2121 (2015).

10. Liu, L. et al. Homo- and heterotypic association regulates signalling by the SgK269/PEAK1 and SgK223 pseudokinases. J. Biol. Chem. 291, 21571-21583 (2016).

11. Fukuda, K., Gupta, S., Chen, K., Wu, C. \& Qin, J. The pseudoactive site of ILK is essential for its binding to alpha-Parvin and localization to focal adhesions. Mol. Cell 36, 819-830 (2009).

12. Murphy, J. M. et al. A robust methodology to subclassify pseudokinases based on their nucleotide-binding properties. Biochem. J. 457, 323-334 (2014).

13. Scheeff, E. D., Eswaran, J., Bunkoczi, G., Knapp, S. \& Manning, G. Structure of the pseudokinase VRK3 reveals a degraded catalytic site, a highly conserved kinase fold, and a putative regulatory binding site. Structure 17, 128-138 (2009).

14. Safari, F., Murata-Kamiya, N., Saito, Y. \& Hatakeyama, M. Mammalian Pragmin regulates Src family kinases via the Glu-Pro-Ile-Tyr-Ala (EPIYA) motif that is exploited by bacterial effectors. Proc. Natl Acad. Sci. USA 108, 14938-14943 (2011)

15. Wang, Y. et al. Pseudopodium-enriched atypical kinase 1 regulates the cytoskeleton and cancer progression [corrected]. Proc. Natl Acad. Sci. USA 107, 10920-10925 (2010).

16. Zheng, Y. et al. Temporal regulation of EGF signalling networks by the scaffold protein Shc1. Nature 499, 166-171 (2013).

17. Tanaka, H., Katoh, H. \& Negishi, M. Pragmin, a novel effector of Rnd2 GTPase, stimulates RhoA activity. J. Biol. Chem. 281, 10355-10364 (2006).

18. Senda, Y., Murata-Kamiya, N. \& Hatakeyama, M. C-terminal Src kinasemediated EPIYA phosphorylation of Pragmin creates a feed-forward Cterminal Src kinase activation loop that promotes cell motility. Cancer Sci. 107, 972-980 (2016)

19. Tactacan, C. M. et al. The pseudokinase SgK223 promotes invasion of pancreatic ductal epithelial cells through JAK1/Stat3 signaling. Mol. Cancer 14, 139 (2015).

20. Leroy, C. et al. Quantitative phosphoproteomics reveals a cluster of tyrosine kinases that mediates SRC invasive activity in advanced colon carcinoma cells. Cancer Res. 69, 2279-2286 (2009).

21. Bristow, J. M., Reno, T. A., Jo, M., Gonias, S. L. \& Klemke, R. L. Dynamic phosphorylation of tyrosine 665 in pseudopodium-enriched atypical kinase 1 (PEAK1) is essential for the regulation of cell migration and focal adhesion turnover. J. Biol. Chem. 288, 123-131 (2013).
22. Croucher, D. R. et al. Involvement of Lyn and the atypical kinase SgK269/ PEAK1 in a basal breast cancer signaling pathway. Cancer Res. 73, 1969-1980 (2013).

23. Kelber, J. A. et al. KRas induces a Src/PEAK1/ErbB2 kinase amplification loop that drives metastatic growth and therapy resistance in pancreatic cancer. Cancer Res. 72, 2554-2564 (2012).

24. Donnelly, N., Gorman, A. M., Gupta, S. \& Samali, A. The eIF2alpha kinases: their structures and functions. Cell Mol. Life Sci. 70, 3493-3511 (2013).

25. Taylor, S. S. \& Kornev, A. P. Protein kinases: evolution of dynamic regulatory proteins. Trends Biochem. Sci. 36, 65-77 (2011).

26. Labesse, G. et al. ROP2 from Toxoplasma gondii: a virulence factor with a protein-kinase fold and no enzymatic activity. Structure 17, 139-146 (2009).

27. Artim, S. C., Mendrola, J. M. \& Lemmon, M. A. Assessing the range of kinase autoinhibition mechanisms in the insulin receptor family. Biochem. J. 448, 213-220 (2012).

28. Hildebrand, J. M. et al. Activation of the pseudokinase MLKL unleashes the four-helix bundle domain to induce membrane localization and necroptotic cell death. Proc. Natl Acad. Sci. USA 111, 15072-15077 (2014).

29. Jonas, S. et al. An asymmetric PAN3 dimer recruits a single PAN2 exonuclease to mediate mRNA deadenylation and decay. Nat. Struct. Mol. Biol. 21, 599-608 (2014).

30. Schafer, I. B., Rode, M., Bonneau, F., Schussler, S. \& Conti, E. The structure of the Pan2-Pan3 core complex reveals cross-talk between deadenylase and pseudokinase. Nat. Struct. Mol. Biol. 21, 591-598 (2014).

31. Kim, C., Xuong, N. H. \& Taylor, S. S. Crystal structure of a complex between the catalytic and regulatory (RIalpha) subunits of PKA. Science 307, 690-696 (2005).

32. Studier, F. W. Protein production by auto-induction in high density shaking cultures. Protein Expr. Purif. 41, 207-234 (2005).

33. Sreenath, A. S. et al. Evidence for the association of synaptotagmin with glutathione S-transferases: implications for a novel function in human breast cancer. Clin. Biochem. 38, 436-443 (2005).

34. Kabsch, W. XDS. Acta Crystallogr. D Biol. Crystallogr. 66, 125-132 (2010).

35. Kabsch, W. Integration, scaling, space-group assignment and post-refinement. Acta Crystallogr. D Biol. Crystallogr. 66, 133-144 (2010).

36. Panjikar, S., Parthasarathy, V., Lamzin, V. S., Weiss, M. S. \& Tucker, P. A. Auto-rickshaw: an automated crystal structure determination platform as an efficient tool for the validation of an X-ray diffraction experiment. Acta Crystallogr. D Biol. Crystallogr. 61, 449-457 (2005).

37. Collaborative Computational Project, N. The CCP4 suite: programs for protein crystallography. Acta Crystallogr. D Biol. Crystallogr. 50, 760-763 (1994).

38. Schneider, T. R. \& Sheldrick, G. M. Substructure solution with SHELXD. Acta Crystallogr. D Biol. Crystallogr. 58, 1772-1779 (2002).

39. Hao, Q. ABS: a program to determine absolute configuration and evaluate anomalous scatterer substructure. J. Appl. Crystallogr. 37, 498-499 (2004).

40. Pannu, N. S., McCoy, A. J. \& Read, R. J. Application of the complex multivariate normal distribution to crystallographic methods with insights into multiple isomorphous replacement phasing. Acta Crystallogr. D Biol. Crystallogr. 59, 1801-1808 (2003).

41. Terwilliger, T. C. Maximum-likelihood density modification. Acta Crystallogr. D Biol. Crystallogr. 56, 965-972 (2000).

42. Cowtan, K. The Buccaneer software for automated model building. 1. Tracing protein chains. Acta Crystallogr. D Biol. Crystallogr. 62, 1002-1011 (2006).

43. Panjikar, S., Parthasarathy, V., Lamzin, V. S., Weiss, M. S. \& Tucker, P. A. On the combination of molecular replacement and single-wavelength anomalous diffraction phasing for automated structure determination. Acta Crystallogr. D Biol. Crystallogr. 65, 1089-1097 (2009).

44. Emsley, P. \& Cowtan, K. Coot: model-building tools for molecular graphics. Acta Crystallogr. D Biol. Crystallogr. 60, 2126-2132 (2004).

45. Afonine, P. V. et al. Towards automated crystallographic structure refinement with phenix.refine. Acta Crystallogr. D Biol. Crystallogr. 68, 352-367 (2012).

46. Davis, I. W. et al. MolProbity: all-atom contacts and structure validation for proteins and nucleic acids. Nucleic Acids Res. 35, W375-W383 (2007).

47. Krissinel, E. \& Henrick, K. Inference of macromolecular assemblies from crystalline state. J. Mol. Biol. 372, 774-797 (2007).

48. Goujon, M. et al. A new bioinformatics analysis tools framework at EMBL-EBI. Nucleic Acids Res. 38, W695-W699 (2010).

49. Sievers, F. et al. Fast, scalable generation of high-quality protein multiple sequence alignments using Clustal Omega. Mol. Syst. Biol. 7, 539 (2011).

50. Murphy, J. M. et al. Insights into the evolution of divergent nucleotide-binding mechanisms among pseudokinases revealed by crystal structures of human and mouse MLKL. Biochem. J. 457, 369-377 (2014).

51. Schuck, P. Size-distribution analysis of macromolecules by sedimentation velocity ultracentrifugation and lamm equation modeling. Biophys. J. 78, 1606-1619 (2000). 
52. Laue, T. M., Shah, B. D., Ridgeway, T. M. \& Pelletier, S. L. Analytical Ultracentrifugation in Biochemistry and Polymer Science (eds Harding, S. E. Rowe, A. J. \& Horton, J. C.) (Royal Society of Chemistry, Cambridge, UK, 1992).

53. Kershaw, N. J. et al. SOCS3 binds specific receptor-JAK complexes to control cytokine signaling by direct kinase inhibition. Nat. Struct. Mol. Biol. 20, 469-476 (2013).

54. Varghese, L. N. et al. Mechanistic insights into activation and SOCS3-mediated inhibition of myeloproliferative neoplasm-associated JAK2 mutants from biochemical and structural analyses. Biochem. J. 458, 395-405 (2014).

55 . Kirby, N. et al. Improved radiation dose efficiency in solution SAXS using a sheath flow sample environment. Acta Crystallogr. D Struct. Biol. 72, 1254-1266 (2016)

56. Konarev, P. V., Volkov, V. V., Sokolova, A. V., Koch, M. H. J. \& Svergun, D. I. PRIMUS: a Windows PC-based system for small-angle scattering data analysis. J. Appl. Crystallogr. 36, 1277-1282 (2003).

57. Svergun, D. I. Determination of the regularization parameter in indirect-transform methods using perceptual criteria. J. Appl. Crystallogr. 25, 495-503 (1992).

58. Svergun, D. I. B., Barberato, C. \& Koch, M. H. J. CRYSOL-a program to evaluate X-ray solution scattering of biological macromolecules from atomic coordinates. J. Appl. Crystallogr. 28, 768-773 (1995).

59. Geback, T., Schulz, M. M., Koumoutsakos, P. \& Detmar, M. TScratch: a novel and simple software tool for automated analysis of monolayer wound healing assays. Biotechniques 46, 265-274 (2009).

60. Jonkman, J. E. et al. An introduction to the wound healing assay using live-cell microscopy. Cell Adh. Migr. 8, 440-451 (2014).

\section{Acknowledgements}

O.P. and M.D.W.G. were supported by ARC Future Fellowships (FT120100056 and FT140100544, respectively). J.M.M. and R.J.D. were supported by NHMRC Fellowships (1105754 and 1058540, respectively). Additional support was received from the Australian Cancer Research Foundation (to I.S.L., W.D., O.P., A.K. and C.Z.). I.S.L. acknowledges support from the Walter and Eliza Hall Institute of Medical Research. This work was also supported by funds from the Operational Infrastructure Support Program provided by the Victorian Government, Australia and NHMRC IRIISS 9000220, and in part by a Project Grant (1084621) from the NHMRC (to R.J.D.). Crystallization experiments were performed at CSIRO Collaborative Crystallization Centre (www.csiro. au/C3), Melbourne, Australia. We thank MX and SAXS beamline staff at the Australian Synchrotron where diffraction and scattering data were collected. We acknowledge the facilities, scientific and technical assistance of Monash Micro Imaging, Monash University, Victoria, Australia and Mandy Magias for technical assistance.

\section{Author contributions}

Conceptualization O.P., M.D.W.G., R.J.D. and I.S.L.; methodology M.D.W.G., S.P., W.D., C.Z., J.M.M., A.K., X.M. and H.C.; investigation W.D., S.P., C.Z., M.D.W.G., O.P. and J.M.M.; writing original to final draft O.P. and I.S.L. with input from M.D.W.G., J.M.M and R.J.D.; review and editing M.D.W.G., O.P., J.M.M., R.J.D. and I.S.L.; funding acquisition I.S.L. and R.J.D.; project administration and supervision I.S.L., O.P. and R.J.D.

\section{Additional information}

Supplementary Information accompanies this paper at doi:10.1038/s41467-017-01279-9.

Competing interests: The authors declare no competing financial interests.

Reprints and permission information is available online at http://npg.nature.com/ reprintsandpermissions/

Publisher's note: Springer Nature remains neutral with regard to jurisdictional claims in published maps and institutional affiliations.

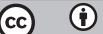

Open Access This article is licensed under a Creative Commons Attribution 4.0 International License, which permits use, sharing, adaptation, distribution and reproduction in any medium or format, as long as you give appropriate credit to the original author(s) and the source, provide a link to the Creative Commons license, and indicate if changes were made. The images or other third party material in this article are included in the article's Creative Commons license, unless indicated otherwise in a credit line to the material. If material is not included in the article's Creative Commons license and your intended use is not permitted by statutory regulation or exceeds the permitted use, you will need to obtain permission directly from the copyright holder. To view a copy of this license, visit http://creativecommons.org/ licenses/by/4.0/.

(C) The Author(s) 2017 